Pacific

Journal of

Mathematics

AREA-MINIMIZING REGIONS WITH SMALL VOLUME IN RIEMANNIAN MANIFOLDS WITH BOUNDARY

Mouhamed Moustapha Fall 


\title{
AREA-MINIMIZING REGIONS WITH SMALL VOLUME IN RIEMANNIAN MANIFOLDS WITH BOUNDARY
}

\author{
Mouhamed Moustapha Fall
}

\begin{abstract}
Given a domain $\Omega$ of a Riemannian manifold, we prove that regions minimizing the area (relative to $\Omega$ ) are nearly the maxima of the mean curvature of $\partial \Omega$ when their volume tends to zero. We deduce some sharp local relative isoperimetric inequalities involving mean curvature comparisons.
\end{abstract}

\section{Introduction}

Let $\Omega$ be a bounded smooth domain of a Riemannian manifold $\left(\mu^{n+1}, g\right)$. Recall that the partitioning problem in $\Omega$ consists in finding, for a given $v<|\Omega|_{g}$, a critical point of the perimeter functional $\mathscr{P}_{g}(\cdot, \Omega)$ in the class of Borel sets in $\Omega$ that enclose a volume $v$. A set that minimizes the perimeter will be called an isoperimetric region. It is clear that the boundary of a smooth solution to the partitioning problem in $\Omega$ have constant mean curvature and, if it touches $\partial \Omega$, it will intersect it orthogonally; see for example [Ros and Vergasta 1995]. In light of standard results in geometric measure theory, minimizers do exist for any given volume and may have various topologies; see the survey [Ros 2005].

Up to now the complete description of minimizers has been achieved only in special cases; see for example [Bürger and Kuwert 2008; Ros and Vergasta 1995; Ritoré and Rosales 2004; Sternberg and Zumbrun 1998]. However, the study of existence and geometric and topological properties of stationary surfaces (not necessarily minimizers) is far from complete. Grüter and Jost [1986] have proved the existence of minimal discs in convex bodies, while Jost [1986] proved the existence of embedded minimal surfaces of higher genus. In the particular case of the free boundary Plateau problem, some global existence results were obtained by M. Struwe [1984; 1988]. In [Fall 2007] we proved the existence of surfaces similar to half spheres surrounding a small volume near nondegenerate critical points of the mean curvature of $\partial \Omega$; in the same paper it was shown that the boundary mean

MSC2000: 53A10, 53C20, 53C21.

Keywords: CMC surfaces, constant mean curvature, isoperimetric profile, isoperimetric region.

Supported by M.U.R.S.T. within the PRIN 2006 "Variational Methods and Nonlinear Differential Equations". 
curvature determines the main terms when studying the problem via a LyapunovSchmidt reduction. In Appendix B we complement this last result as follows:

Proposition B.1. There exist $r_{0}>0$ and a smooth function $f:\left(0, r_{0}\right) \times \partial \Omega \rightarrow \mathbb{R}$ such that for every $r \in\left(0, r_{0}\right)$, if $p$ is a critical point of $f(r, \cdot)$, the geodesic ball centered at $p$ with radius $r$ can be perturbed smoothly to a set which is a solution to the partitioning problem in $\Omega$. Furthermore

$$
\left\|f(r, \cdot)-H_{\partial \Omega}(\cdot)\right\|_{\mathscr{C}^{1}(\partial \Omega)} \leq c r,
$$

where $H_{\partial \Omega}(p)$ is the mean curvature of $\partial \Omega$ at $p$.

In the body of the paper our main goal is the location of minimal area separating hypersurfaces of $\Omega$ enclosing a small volume:

Theorem 1.2. Isoperimetric regions with small volume in $\Omega$ are near global maxima of the mean curvature of $\partial \Omega$.

Results of this kind were recently obtained in [Druet 2002; Nardulli 2007]. These authors showed that isoperimetric regions with small volume are nearly maxima of the scalar curvature.

To prove the above theorem, we first show a regularity result that generalizes Theorem 2.2 in [Morgan and Johnson 2000] (see Lemma 4.2). We notice that the original proof of that theorem highlights that the diameter of an isoperimetric region $E_{v}$ tends to zero as the volume $v$ tends to zero. Moreover as pointed out by Bayle and Rosales [2005], this set must touch the boundary $\partial \Omega$ if $v$ is small enough ( $E_{v}$ is not compactly contained in $\Omega$ ). From this one sees that $E_{v}$ is contained in a geodesic sphere centered at some point $p \in \partial \Omega$ for $v$ small. Hence, using the result of Morgan and Johnson just mentioned, one concludes that the hypersurface $\Sigma_{v}=\partial E_{v} \cap \Omega$ can be written, after suitable scaling, as a graph over a round hemisphere, and the function defining the graph tends to zero. This also shows that $\partial \Sigma_{v} \subset \partial \Omega$. But, according to our argument we need convergence up to the free boundary. We achieve this, following [Grüter 1987], by proving a monotonicity result for the area of $\Sigma_{v}$ in a tubular neighborhood of $\partial \Omega$. This allows us to get a bound for the area of $\partial \Sigma_{v}$ and hence, by compactness, weak convergence up to the free boundary and smoothly by [Grüter and Jost 1986].

The second step is to reduce the isoperimetric problem to a finite-dimensional variational one (see Lemma 4.7) by adopting a variant of the method in [Nardulli 2007]. To this end, by means of the implicit function theorem we construct, for any fixed $v$ sufficiently small, a manifold $\mathfrak{C}_{v}$ of sets having volume $v$ and diffeomorphic to $\partial \Omega$ (see Lemma 4.6). Any set $E \in \mathfrak{C}_{v}$ is a pseudo-half-ball (see Definition 4.5) which is uniquely determined by its center of mass $p \in \partial \Omega$ while its boundary, $\partial E=\Sigma_{p, \omega^{p, v}}$, is a normal graph over a geodesic sphere centered at $p$ with $\omega^{p, v}$ (defining the graph) tending to zero as $v \rightarrow 0$. Finally we show that an 
isoperimetric region with small volume $v$ must belong to $\mathfrak{C}_{v}$, so looking for the minimum of the perimeter among sets in $\Omega$ with volume $v$ is equivalent to taking the minimum among sets in $\mathfrak{C}_{v}$. Taking advantage of the role of the mean curvature in the expansion of the area of normal graphs centered at the free boundary $\partial \Omega$ (see Appendix A), the theorem then follows.

It is well known that much of the information concerning the partitioning problem is contained in the isoperimetric profile relative to $\Omega$, namely the mapping

$$
v \mapsto I_{\Omega}(v)=\min _{\substack{E \subset \Omega \\|E|_{g}=v}} \mathscr{P}_{g}(E, \Omega) .
$$

Explicit lower bounds for the profile $I_{\Omega}$ are very important in applications and are called isoperimetric inequalities; for instance see [Chavel 1984; 2001].

Nardulli [2007, Theorem 7] gave an expansion of the isoperimetric profile of a compact Riemannian manifold $\mathcal{M}^{n+1}$ as

$$
I_{\mathcal{M}}(v)=\left(1-\gamma_{n} \max _{p \in \mathcal{M}} S_{\mathcal{M}}(p) v^{2 /(n+1)}+O\left(v^{4 /(n+1)}\right)\right) I_{\mathbb{R}^{n+1}}(v),
$$

where $S_{\mathcal{M}}$ is the scalar curvature of $\mathcal{M}$ and $\gamma_{n}$ is a positive number. This was obtained after showing that small isoperimetric regions can be written as normal graphs over the boundary of a geodesic ball $B_{\mu}(p, r)$ centered at some point $p$. So he reduced the isoperimetric problem to a finite-dimensional one.

In our situation, as we will see later, the presence of the obstacle $\partial \Omega$ is not negligible and in fact the second fundamental form of $\partial \Omega$ determines the first-order expansion of the profile $I_{\Omega}$. Before going on, we recall that Bayle and Rosales [2005] have shown that

$$
I_{\Omega}(v)=(1+O(v)) I_{\mathbb{R}_{+}^{n+1}}(v),
$$

where $I_{\mathbb{R}_{+}^{n+1}}(v)=(n+1)\left|B_{+}^{n+1}\right|^{1 /(n+1)} v^{n /(n+1)}$. From the reduction of the isoperimetric problem to a finite-dimensional one (Lemma 4.7), we can determine the first coefficient of the asymptotic expansions of the profile of $\Omega$ near zero. Letting $v=\left|r B_{+}^{n+1}\right|$ in Lemmas 4.7 and 4.8, we show that

$$
I_{\Omega}(v)=\min _{p \in \partial \Omega}\left\{I_{\mathbb{B}_{+}^{n+1}}(v)-\frac{n}{n+2} \frac{\left|B^{n}\right|}{\left|B_{+}^{n+1}\right|} H_{\partial \Omega}(p) v+O_{p}\left(v^{(n+2) /(n+1)}\right)\right\},
$$

where $H_{\partial \Omega}(p)$ is the mean curvature of $\partial \Omega$ at $p$ and $O_{p}(\rho)$ is a smooth function in $p$ and $\rho$ tending to zero uniformly with respect to $p$ as $\rho$ tends to zero. Hence:

Corollary 1.3. With $\beta_{n}=\frac{n}{(n+1)(n+2)} \frac{\left|B^{n}\right|}{\left|B_{+}^{n+1}\right|^{(n+2) /(n+1)}}$, we have

$$
I_{\Omega}(v) \sim\left(1-\beta_{n} \max _{p \in \partial \Omega} H_{\partial \Omega}(p) v^{1 /(n+1)}+O\left(v^{2 /(n+1)}\right)\right) I_{\mathbb{R}_{+}^{n+1}}(v) .
$$


Choe, Ghomi and Ritore [2007] showed that an isoperimetric region outside a convex domain in Euclidean space has no less perimeter than the area of a hemisphere, provided it encloses the volume of a half-ball. In this situation, from Corollary 1.3 , we can weaken convexity by strict H-convexity (that is, the domain has nonnegative mean curvature).

Corollary 1.4. If $\Omega$ is a strictly $H$-convex smooth bounded domain of $\mathbb{R}^{n+1}$ and $v$ is small enough,

$$
I_{\mathbb{R}^{n+1} \backslash \Omega}(v)>I_{\mathbb{R}_{+}^{n+1}}(v) .
$$

This, therefore, moves toward the question of isoperimetric inequalities inside $\mathrm{H}$ convex domains.

Druet [2002] proved a local sharp isoperimetric inequality, with the aim of answering a question from [Morgan and Johnson 2000]: In a compact Riemannian manifold $\left(\mathcal{M}^{n+1}, g\right)$ whose scalar curvature satisfies $\max _{p \in \partial \mu} S_{g}(p)<n(n+1) K_{0}$ for some $K_{0} \in \mathbb{R}$, the perimeter of the solution to the isoperimetric problem with small volume is strictly greater than that of an isoperimetric region in the space form of constant sectional curvature $K_{0}$. In this direction we have obtained:

Corollary 1.5. Suppose $\Omega$ is a bounded smooth domain in a Riemannian manifold $\left(\mu^{n+1}, g\right)$. Let $\Omega_{0}$ be a bounded smooth domain in any other Riemannian manifold $\left(\mu_{0}^{n+1}, g_{0}\right)$ with mean curvatures satisfying $\max _{p \in \partial \Omega} H_{\partial \Omega}(p)<\max _{p \in \partial \Omega_{0}} H_{\partial \Omega_{0}}$. Then, if $v$ is small enough,

$$
I_{\Omega}(v)>I_{\Omega_{0}}(v) .
$$

\section{Preliminaries}

We denote by $N_{\partial \Omega}$ the unit interior normal vector field along $\partial \Omega$. We consider an oriented orthonormal frame field $\left(E_{1}, \ldots, E_{n}, N_{\partial \Omega}\right)$ of $\mathcal{M}$ along $\partial \Omega$, and use it to introduce geodesic normal coordinates in a neighborhood (in $\partial \Omega$ ) of a point $p \in \partial \Omega$ with coordinates $x^{\prime}=\left(x^{1}, \ldots, x^{n}\right) \in \mathbb{R}^{n}$. We set

$$
f^{p}\left(x^{\prime}\right):=\exp _{p}^{\partial \Omega}\left(x^{i} E_{i}\right) .
$$

This choice of coordinates induces coordinate vector fields on $\partial \Omega$ :

$$
Y_{i}\left(x^{\prime}\right)=f_{*}\left(\partial_{x^{i}}\right), \quad \text { for } i=1, \ldots, n .
$$

For any vector field $Y$ on $T \partial \Omega$, we define $S(Y)=\nabla_{Y} N_{\partial \Omega}$, where $\nabla$ is the connection on $M$.

Now consider a local parametrization of a neighborhood of $p$ in $\mu$ by

$$
F^{p}(x):=\exp _{f^{p}\left(x^{\prime}\right)}^{\mu}\left(x^{n+1} N_{\partial \Omega}\right), \quad x=\left(x^{\prime}, x^{n+1}\right) \in \mathbb{R}^{n+1} .
$$


This yields the coordinate vector fields in $M$

$$
\begin{aligned}
X_{i}(x) & :=F_{*}^{p}\left(\partial_{x^{i}}\right), \quad i=1, \ldots, n, \\
X_{n+1}(x) & :=F_{*}^{p}\left(\partial_{x^{n+1}}\right) .
\end{aligned}
$$

In the whole paper, for any vector fields $X, Y \in T M$, we define $\langle X, Y\rangle:=g(X, Y)$. We denote by $R_{p}$ and $R_{p}^{\mu}$ the Riemannian tensors of $\partial \Omega$ and $M$, respectively.

Lemma 2.1. Near the point $F^{p}\left(x^{\prime}, 0\right)=f^{p}\left(x^{\prime}\right)$,

$$
X_{i}=Y_{i}+x^{n+1} S\left(Y_{i}\right)+\frac{1}{2}\left(x^{n+1}\right)^{2} R_{p}^{\mu}\left(N_{\partial \Omega}, Y_{i}\right) N_{\partial \Omega}+O\left(\left|x^{n+1}\right|^{3}\right) .
$$

Near $p=F^{p}(0)$ we have

$$
\left\langle Y_{i}, Y_{j}\right\rangle=\delta_{i j}+\frac{1}{3}\left\langle R_{p}\left(E_{k}, E_{i}\right) E_{l}, E_{j}\right\rangle x^{k} x^{l}+O\left(|x|^{3}\right) .
$$

Proof. By construction,

$$
\left.\nabla_{X_{n+1}}^{k} X_{n+1}\right|_{f\left(x^{\prime}\right)}=0 \quad \text { for any integer } k \geq 1 .
$$

Now since $\left.\nabla_{X_{n+1}} X_{i}\right|_{f\left(x^{\prime}\right)}=\left.\nabla_{X_{i}} X_{n+1}\right|_{f\left(x^{\prime}\right)}=S\left(Y_{i}\right)$ and $\left.X_{n+1}\right|_{f\left(x^{\prime}\right)}=N_{\partial \Omega}$, we get

$$
\begin{aligned}
\left.\nabla_{X_{n+1}}^{2} X_{i}\right|_{f\left(x^{\prime}\right)} & =\left.\nabla_{X_{n+1}}\left(\nabla_{X_{i}} X_{n+1}\right)\right|_{f\left(x^{\prime}\right)} \\
& =R_{p}^{\mu}\left(N_{\partial \Omega}, Y_{i}\right) N_{\partial \Omega}+\left.\nabla_{X_{i}} \nabla_{X_{n+1}} X_{n+1}\right|_{f\left(x^{\prime}\right)}=R_{p}^{\mu}\left(N_{\partial \Omega}, Y_{i}\right) N_{\partial \Omega} .
\end{aligned}
$$

For the proof of the last expansions, see for example [Pacard and Xu 2009, Proposition 2.1].

This lemma affords the next proposition, which gives expansions of the metric $g_{\alpha \beta}:=\left\langle X_{\alpha}, X_{\beta}\right\rangle$ in a neighborhood of $p \in \partial \Omega$ in $\mathcal{M}$ with $\alpha, \beta \in\{1, \ldots, n, n+1\}$.

Proposition 2.2. In a neighborhood of $p$,

$$
\begin{gathered}
g_{i j}=\delta_{i j}+2\left\langle S\left(Y_{i}\right), Y_{j}\right\rangle x^{n+1}+\frac{1}{3}\left\langle R_{p}\left(E_{k}, E_{i}\right) E_{l}, E_{j}\right\rangle x^{k} x^{l} \\
+\left(\left\langle R_{p}^{\mu}\left(N_{\partial \Omega}, E_{i}\right) N_{\partial \Omega}, E_{j}\right\rangle+\left\langle S\left(Y_{i}\right), S\left(Y_{j}\right)\right\rangle\right)\left(x^{n+1}\right)^{2}+O\left(|x|^{3}\right), \\
g_{i n+1}=O\left(|x|^{3}\right), \quad g_{n+1 n+1}=1 .
\end{gathered}
$$

We fix the following notation:

$$
\mathbb{R}_{+}^{n+1}:=\left\{x=\left(x^{1}, \ldots, x^{n+1}\right) \in \mathbb{R}^{n+1}: x^{n+1}>0\right\} .
$$

Let $B^{n+1}$ be the unit ball of $\mathbb{R}^{n+1}$ centered at the origin. We define

$$
B_{+}^{n+1}=B^{n+1} \cap \mathbb{R}_{+}^{n} \quad \text { and } \quad S_{+}^{n}:=\partial B_{+}^{n+1} .
$$

We will denote by $\Theta: B^{n} \rightarrow S_{+}^{n}$ the inverse of the stereographic projection from the south pole. $\Theta=\left(\Theta^{1}, \ldots, \Theta^{n}, \Theta^{n+1}\right)$ is a conformal parametrization of $S_{+}^{n}$ 
and, for any $z=\left(z^{1}, \ldots, z^{n}\right) \in B^{n}$,

$$
\Theta(z)=(z \mu(z), \mu(z)-1)=\left(\frac{2 z^{1}}{1+|z|^{2}}, \ldots, \frac{2 z^{n}}{1+|z|^{2}}, \frac{1-|z|^{2}}{1+|z|^{2}}\right)
$$

with conformal factor given by

$$
\mu(z):=\frac{2}{1+|z|^{2}} .
$$

We often use the projection of $\Theta$ on $\mathbb{R}^{n} \times\{0\}$ and denote it by

$$
\widetilde{\Theta}(z):=(z, 0) \mu(z) .
$$

The next lemma collect some useful properties of the function $\Theta$. We omit the proof, which can be obtained with elementary computations.

Lemma 2.3. For every $i, j, l=1, \ldots, n$,

$$
\begin{aligned}
& \Theta_{i}^{n+1}=-\mu \Theta^{i}, \\
& \widetilde{\Theta}_{i}=-\Theta^{i} \widetilde{\Theta}+\mu E_{i}, \\
& \left\langle\Theta_{i}, \Theta_{j}\right\rangle=\mu^{2} \delta_{i j}, \\
& \Delta_{S^{n}}=\Delta_{\mathbb{R}^{n}}-\left\langle\Theta_{i i}, \Theta_{k}\right\rangle \Theta^{k} \partial_{k} .
\end{aligned}
$$

Here $\Theta_{l}$ is the partial derivative of $\Theta$ in the variable $z^{l}$.

Observe that all hypersurfaces nearby a geodesic sphere centered at $p \in \partial \Omega$ with radius $r$ can be parametrized by a mapping $G: B^{n} \rightarrow \mathcal{M}$ defined by

$$
G(z):=F^{p}\left(r(1+\omega) \widetilde{\Theta}(z), r(1+\omega) \Theta^{n+1}(z)\right),
$$

for some $p \in \partial \Omega$ and $\omega: S_{+}^{n} \rightarrow \mathbb{R}$. By construction, since $\Theta^{n+1}=0$ on $\partial S_{+}^{n}$,

$$
\partial \Sigma_{p, r, \omega} \subset \partial \Omega \text {. }
$$

Given $p \in \partial \Omega$ and $\omega: S_{+}^{n} \rightarrow \mathbb{R}$, throughout this paper, the expression $\Sigma_{p, r, \omega}$ will denote the hypersurface $F^{p}\left(r(1+\omega) S_{+}^{n}\right)$, while $E_{p, r, \omega}$ will denote the set bounded by $\Sigma_{p, r, \omega}$ and $\partial \Omega$.

Notation. Any expression of the form $L_{p}(\omega)$ denotes a linear combination of the function $\omega$ together with its derivatives of order up to 2 with respect to the vector fields $\Theta_{i}$. Similarly, $\bar{L}_{p}(\omega)$ will denote such a linear combination with first derivatives. The coefficients of $L_{p}$ or $\bar{L}_{p}$ might depend on $r$ and $p$ but, for all $k \in \mathbb{N}$, there exists a constant $c>0$ independent of $r \in(0,1)$ and $p \in \partial \Omega$ such that

$$
\left\|L_{p}(\omega)\right\|_{\mathscr{C}^{k, \alpha}\left(\overline{S_{+}^{n}}\right)} \leq c\|\omega\|_{\mathscr{C}^{k+2, \alpha}\left(\overline{S_{+}^{n}}\right)}, \quad\left\|\bar{L}_{p}(\omega)\right\|_{\mathscr{C}^{k, \alpha}\left(\overline{S_{+}^{n}}\right)} \leq c\|\omega\|_{\mathscr{C}^{k+1, \alpha}\left(\overline{S_{+}^{n}}\right)} .
$$

Given $a \in \mathbb{N}$, an expression of the form $Q_{p}^{a}(\omega)$ (respectively, $\bar{Q}_{p}^{a}(\omega)$ ) will denote a nonlinear operator in the function $\omega$ together with its $\Theta_{i}$-derivatives of order up to 2 (respectively, order 1). The coefficients of the Taylor expansion of $Q_{p}^{a}(\omega)$ in powers of $\omega$ and its partial derivatives might depend on $r$ and $p$ and, given 
$k \in \mathbb{N}$, there exists a constant $c>0$ independent of $r \in(0,1)$ and $p \in \mathcal{M}$ such that $Q_{p}^{a}(0)=0$ and

$$
\begin{aligned}
& \left\|Q_{p}^{a}\left(\omega_{1}\right)-Q_{p}^{a}\left(\omega_{2}\right)\right\|_{\mathscr{C}^{k, \alpha}\left(\overline{S_{+}^{n}}\right)} \\
& \quad \leq c\left(\left\|\omega_{1}\right\|_{\mathscr{C}^{k+2, \alpha}\left(\overline{S_{+}^{n}}\right)}+\left\|\omega_{2}\right\|_{\mathscr{C}^{k+2, \alpha}\left(\overline{S_{+}^{n}}\right)}\right)^{a-1}\left\|\omega_{1}-\omega_{2}\right\|_{\mathscr{C}^{k+2, \alpha}\left(\overline{S_{+}^{n}}\right)},
\end{aligned}
$$

provided $\left\|\omega_{i}\right\|_{\mathscr{C}^{k+2, \alpha}\left(\overline{S_{+}^{n}}\right)} \leq 1$. Also

$$
\begin{aligned}
& \left\|\bar{Q}_{p}^{a}\left(\omega_{1}\right)-\bar{Q}_{p}^{a}\left(\omega_{2}\right)\right\|_{\mathscr{C}^{k, \alpha}\left(\overline{S_{+}^{n}}\right)} \\
& \quad \leq c\left(\left\|\omega_{1}\right\|_{\mathscr{C}^{k+1, \alpha}\left(\overline{S_{+}^{n}}\right)}+\left\|\omega_{2}\right\|_{\mathscr{C}^{k+1, \alpha}\left(\overline{S_{+}^{n}}\right)}\right)^{a-1}\left\|\omega_{1}-\omega_{2}\right\|_{\mathscr{C}^{k+1, \alpha}\left(\overline{S_{+}^{n}}\right)},
\end{aligned}
$$

provided $\left\|\omega_{i}\right\|_{\mathscr{C}^{k+2, \alpha}\left(\overline{S_{+}^{n}}\right)} \leq 1$. We also agree that any term denoted by $\mathcal{O}_{p}\left(r^{d}\right)$ is a smooth function on $S_{+}^{n}$ that might depend on $p$ but satisfies

$$
\left\|0_{p}\left(r^{d}\right)\right\|_{\mathscr{C}^{k, \alpha}\left(\overline{S_{+}^{n}}\right)} \leq c r^{d},
$$

for a constant $\mathrm{c}$ independent of $p$.

\section{Mean curvature expansion of $\Sigma_{p, r, \omega}$}

We now develop the expansion of the mean curvature $H(p, r, \omega)$ of a hypersurface $\Sigma_{p, r, \omega}$ in terms of $r$ and $\omega$. The proof is similar to the one in [Fall and Mahmoudi 2008], and we just sketch it for the reader's convenience.

Let $z \mapsto G(z)$ parametrize $\Sigma_{p, r, \omega}$ as defined in (4).

Notation. With an abuse of notation, at the point $p$, we let

$$
\Theta:=\Theta^{j} E_{j}+\Theta^{n+1} N_{\partial \Omega}=\tilde{\Theta}+\Theta^{n+1} N_{\partial \Omega}, \quad \Theta_{i}:=\partial_{z^{i}} \Theta^{j} E_{j}+\partial_{z^{i}} \Theta^{n+1} N_{\partial \Omega},
$$

while at the point $G(z)$, we define the vector fields

$$
\Upsilon:=\Theta^{j} X_{j}+\Theta^{n+1} X_{n+1}=\tilde{\Upsilon}+\Theta^{n+1} X_{n+1}, \quad \Upsilon_{i}:=\partial_{z^{i}} \Theta^{j} X_{j}+\partial_{z_{j}} \Theta^{n+1} X_{n+1} .
$$

We also set

$$
\omega_{j}:=\partial_{z^{j}} \omega, \quad \omega_{i j}:=\partial_{z^{i}} \partial_{z^{j}} \omega .
$$

From the notation, it is clear that the tangent space of $\Sigma_{p, r, \omega}$ is spanned by the vector fields

$$
Z_{j}=G_{*}\left(\partial_{z^{j}}\right)=r(1+\omega) \Upsilon_{j}+r \omega_{j} \Upsilon, \quad j=1, \ldots, n .
$$

Letting $g_{i j}^{\Sigma}:=\left\langle Z_{i}, Z_{j}\right\rangle$ be the first fundamental form of $\Sigma_{p, r, \omega}(=\Sigma)$, we conclude using Proposition A.1 that

$$
(1+\omega)^{-2} r^{-2} g_{i j}^{\Sigma}=\mu^{2}+2 r\left\langle S\left(\widetilde{\Theta}_{j}\right), \widetilde{\Theta}_{i}\right\rangle \Theta^{n+1}+\mathcal{O}\left(r^{2}\right)+r L(\omega)+Q(\omega) .
$$


3.1. The normal vector field. We expand the unit normal to $\Sigma_{p, r, \omega}$. Define the vector field

$$
\tilde{N}_{\Sigma}:=-r \Upsilon+\alpha^{j} Z_{j}
$$

It is the outer normal field (not necessarily unitary) along $\Sigma_{p, r, \omega}$ if we can determine $\alpha^{j}$ so that its tangential components $\left\langle\tilde{N}_{\Sigma}, Z_{j}\right\rangle$ vanish. This leads to a linear system for $\alpha^{j}$.

From (15)-(16) there follows the expansion

$$
\left\langle\Upsilon, Z_{j}\right\rangle=r \omega_{j}+2 r^{2} \Theta^{n+1}\left\langle S(\widetilde{\Theta}), \widetilde{\Theta}_{j}\right\rangle+\mathcal{O}\left(r^{3}\right)+r^{2} L(\omega)+r^{2} Q(\omega),
$$

Using (6) and some algebraic calculations, one obtains

$$
\begin{aligned}
\alpha^{j}\left\langle Z_{j}, Z_{i}\right\rangle & =r\left\langle\Upsilon, Z_{j}\right\rangle \\
& =r^{2}\left(\omega_{i}+2 r \Theta^{n+1}\left\langle S(\widetilde{\Theta}), \widetilde{\Theta}_{i}\right\rangle+\mathcal{O}\left(r^{2}\right)+r L(\omega)+Q(\omega)\right)
\end{aligned}
$$

hence straightforward computations imply that

$$
\alpha^{k}\left\langle\Theta_{i}, \Theta_{k}\right\rangle=\omega_{i}+2 r \Theta^{n+1}\left\langle S(\widetilde{\Theta}), \widetilde{\Theta}_{i}\right\rangle+\mathscr{O}\left(r^{2}\right)+r L(\omega)+Q(\omega) .
$$

Also using (7), we have

$$
\begin{aligned}
\left\langle\tilde{N}_{\Sigma}, \tilde{N}_{\Sigma}\right\rangle & =r^{2}\langle\Upsilon, \Upsilon\rangle-2 r \alpha^{k}\left\langle Z_{k}, \Upsilon\right\rangle+\alpha_{l} \alpha_{k}\left\langle Z_{k}, Z_{l}\right\rangle \\
& =r^{2}\left(1+2 r \Theta^{n+1}\langle S(\widetilde{\Theta}), \widetilde{\Theta}\rangle+\mathcal{O}\left(r^{2}\right)+r L(\omega)+Q(\omega)\right)-\alpha_{l} \alpha_{k}\left\langle Z_{k}, Z_{l}\right\rangle \\
& =r^{2}\left(1+2 r \Theta^{n+1}\langle S(\widetilde{\Theta}), \widetilde{\Theta}\rangle+\mathcal{O}\left(r^{2}\right)+r L(\omega)+Q(\omega)\right) .
\end{aligned}
$$

From this we deduce that

$$
\left|\tilde{N}_{\Sigma}\right|^{-1}=r^{-1}\left(1-r \Theta^{n+1}\langle S(\widetilde{\Theta}), \widetilde{\Theta}\rangle+\mathcal{O}\left(r^{2}\right)+r L(\omega)+Q(\omega)\right) .
$$

Therefore the unit normal can be expanded as

$$
N_{\Sigma}=\frac{\tilde{N}_{\Sigma}}{\left|\tilde{N}_{\Sigma}\right|}=-\left(1-r \Theta^{n+1}\langle S(\widetilde{\Theta}), \widetilde{\Theta}\rangle\right) \Upsilon+\alpha_{k} Z_{k}+\left(\mathcal{O}\left(r^{2}\right)+r L(\omega)+Q(\omega)\right)_{\alpha} X_{\alpha} .
$$

3.2. The second fundamental form. We turn to the expansion of the coefficients of the second fundamental form.

By definition $\nabla_{Z_{i}} Z_{j} \simeq D Z_{j} / d z^{i}$, whence we readily get the expansions

$$
\begin{aligned}
r^{-1} \nabla_{Z_{i}} Z_{j}=\omega_{i j} \Upsilon+\omega_{j} \Upsilon_{i}+\omega_{i} \Upsilon_{j}+(1+\omega) \Upsilon_{i j} & +r \Theta_{i}^{\alpha} \Theta_{j}^{\beta} \nabla_{X_{\alpha}} X_{\beta} \\
& +\left(O\left(r^{2}\right)+r L(\omega)+Q(\omega)\right)_{\alpha} X_{\alpha},
\end{aligned}
$$


so using (15)-(16), we get

$$
\begin{aligned}
r^{-1}\left\langle N_{\Sigma}, \nabla_{Z_{i}} Z_{j}\right\rangle= & -(1+\omega)\left(1-r \Theta^{n+1}\langle S(\widetilde{\Theta}), \widetilde{\Theta}\rangle\right)\left\langle\Upsilon_{i j}, \Upsilon\right\rangle-\omega_{i j}+\alpha^{k}\left\langle\Upsilon_{i j}, \Upsilon_{k}\right\rangle \\
& +r\left(\Theta^{n+1}\left\langle S\left(\widetilde{\Theta}_{i}\right), \widetilde{\Theta}_{j}\right\rangle-\Theta_{i}^{n+1}\left\langle S(\widetilde{\Theta}), \widetilde{\Theta}_{j}\right\rangle-\Theta_{j}^{n+1}\left\langle S(\widetilde{\Theta}), \widetilde{\Theta}_{i}\right\rangle\right) \\
& +\mathcal{O}\left(r^{2}\right)+r L(\omega)+Q(\omega) .
\end{aligned}
$$

Observing that

$$
\begin{aligned}
\left\langle\Upsilon_{i j}, \Upsilon\right\rangle & =\left\langle\Theta_{i j}, \Theta\right\rangle+2 r \Theta^{n+1}\left\langle S(\widetilde{\Theta}), \widetilde{\Theta}_{i j}\right\rangle+\mathcal{O}\left(r^{2}\right)+r L(\omega)+Q(\omega), \\
\left\langle\Upsilon_{i j}, \Upsilon_{k}\right\rangle & =\left\langle\Theta_{i j}, \Theta_{k}\right\rangle+\mathcal{O}(r)+r L(\omega)+Q(\omega),
\end{aligned}
$$

we obtain with a little work:

Proposition 3.1. The second fundamental form of the $\Sigma_{p, r, \omega}$ has the expansion

$$
\begin{aligned}
r^{-1}\left\langle N_{\Sigma}, \nabla_{Z_{i}} Z_{j}\right\rangle= & -\left(1+\omega-r \Theta^{n+1}\langle S(\widetilde{\Theta}), \widetilde{\Theta}\rangle\right)\left\langle\Theta_{i j}, \Theta\right\rangle \\
& -\left(\omega_{i j}+2 r \Theta^{n+1}\left\langle S(\widetilde{\Theta}), \widetilde{\Theta}_{i j}\right\rangle\right)+\alpha^{k}\left\langle\Theta_{i j}, \Theta_{k}\right\rangle \\
& +r\left(\Theta^{n+1}\left\langle S\left(\widetilde{\Theta}_{i}\right), \widetilde{\Theta}_{j}\right\rangle-\Theta_{i}^{n+1}\left\langle S(\widetilde{\Theta}), \widetilde{\Theta}_{j}\right\rangle-\Theta_{j}^{n+1}\left\langle S(\widetilde{\Theta}), \widetilde{\Theta}_{i}\right\rangle\right) \\
& +\mathcal{O}\left(r^{2}\right)+r L(\omega)+Q(\omega) .
\end{aligned}
$$

Let $H(p, r, \omega)$ be the mean curvature of the hypersurface $\Sigma_{p, r, \omega}$. Contracting with the metric, (6), and using also Lemma 2.3, we have:

Proposition 3.2. In the notation above, we have

$$
\begin{aligned}
r H(p, r, \omega)=n-\left(\Delta_{S_{+}^{n}} \omega+n \omega\right)+r \Theta^{n+1}\left((n+3)\langle S(\tilde{\Theta}), \tilde{\Theta}\rangle-\left\langle S\left(E_{i}\right), E_{i}\right\rangle\right) \\
+ \\
+\mathcal{O}\left(r^{2}\right)+r L(\omega)+Q(\omega)
\end{aligned}
$$

in $\Sigma_{p, r, \omega}$ and

$$
\left\langle N_{\partial \Sigma_{p, r, \omega}}^{\Sigma_{p, r, \omega}}, N_{\partial \Sigma_{p, r, \omega}}^{\partial \Omega}\right\rangle=-\frac{\partial \omega}{\partial \eta}+r^{2} \bar{L}(\omega)+\bar{Q}(\omega) \quad \text { on } \partial \Sigma_{p, r, \omega}
$$

where $\eta:=-N_{\partial \Omega}$ is the outer unit normal to $\partial S_{+}^{n}$ and $N_{B}^{A}$ is the normal of $B$ in $A$. Proof. We first determine $N_{\partial \Sigma_{p, r, \omega}}^{\partial \Omega}$. Fix a parametrization $s \mapsto \bar{\Theta}(s) \in S^{n-1}=\partial B^{n}$ of the unit sphere. Noting that $\Theta(\bar{\Theta}(s))=\bar{\Theta}(s)$, we see that the mapping

$$
s \mapsto \bar{G}(s):=f^{p}(r(1+\omega) \bar{\Theta}(s))=F^{p}(r(1+\omega) \bar{\Theta}(s), 0)
$$

parametrizes $\partial \Sigma_{p, r, \omega} \subset \partial \Omega$ and hence its tangent space is spanned by

$$
\bar{Z}_{i}=r(1+\omega) \bar{\Upsilon}_{i}+r \partial_{s^{i}} \omega \bar{\Upsilon}, \quad i=1, \ldots, n-1,
$$

where

Therefore, setting

$$
\bar{\Upsilon}:=\bar{\Theta}^{j} Y_{j}, \quad \bar{\Upsilon}_{i}:=\partial_{s^{i}} \bar{\Theta}^{j} Y_{j}
$$

$$
\tilde{N}_{\partial \Sigma_{p, r, \omega}}^{\partial \Omega}=-r \bar{\Upsilon}+\beta^{k} \bar{Z}_{k}
$$


we need only to find $\beta^{k}, k=1, \ldots, n$ so that it is orthogonal to $\bar{Z}_{i}$. This can be found in [Pacard and Xu 2009, Lemma 2.1] and one has

$$
\beta^{k}\left\langle\bar{Z}_{k}, \bar{Z}_{i}\right\rangle=r \partial_{s^{i}} \omega
$$

while $r^{-2}\left\langle\bar{Z}_{k}, \bar{Z}_{i}\right\rangle=\left\langle\bar{\Theta}_{k}, \bar{\Theta}_{i}\right\rangle\left(1+\mathcal{O}\left(r^{2}\right)+r^{2} L(\omega)+Q(\omega)\right)$ and $\left|\tilde{N}_{\partial \Sigma_{p, r, \omega}}^{\partial \Omega}\right|^{-1}=$ $r^{-1}(1+Q(\omega))$.

We now determine $N_{\partial \Sigma_{p, r, \omega}}^{\Sigma_{p, r, \omega}}$. To this aim, we denote by $v$ the unit outer normal to the unit disc $B^{n}$ and similarly as we have expanded $\tilde{N}_{\partial \Sigma_{p, r, \omega}}^{\partial \Omega}$, we let

$$
\begin{aligned}
\tilde{N}_{\partial \Sigma}^{\Sigma} & =\left.G_{*}\left(\partial_{\nu}\right)\right|_{\partial B_{1}^{n}}+\gamma^{k} \bar{Z}_{k}=-r(1+\omega) N_{\partial \Omega}+\left.r \partial_{\nu} \omega \tilde{\Upsilon}\right|_{\partial B_{1}^{n}}+\gamma^{k} \bar{Z}_{k} \\
& =-r(1+\omega) N_{\partial \Omega}+r \partial_{\nu} \omega \bar{\Upsilon}+\gamma^{k} \bar{Z}_{k} .
\end{aligned}
$$

Here, we have used that $\left.\partial_{\nu} \widetilde{\Theta}\right|_{\partial B_{1}^{n}}=0$ and $\left.\partial_{\nu} \Theta^{n+1}\right|_{\partial B_{1}^{n}}=-1$. Noting that $\left\langle N_{\partial \Omega}, \bar{Z}_{j}\right\rangle$ vanishes and $\left\langle\bar{\Upsilon}, \bar{Z}_{j}\right\rangle=r \omega_{j}$, we see that $\tilde{N}_{\partial \Sigma}^{\Sigma} \in T \Sigma_{p, r, \omega}$ is normal to $\partial \Sigma_{p, r, \omega}$ if

$$
\gamma^{k}\left\langle\bar{Z}_{k}, \bar{Z}_{j}\right\rangle=r^{2} \omega_{j} \partial_{\nu} \omega .
$$

We also deduce that $\left|\tilde{N}_{\partial \Sigma}^{\Sigma}\right|^{-1}=r^{-1}(1+Q(\omega))$.

Collecting these with the fact that $\left\langle N_{\partial \Sigma}^{\Sigma}, N_{\partial \Sigma}^{\partial \Omega}\right\rangle=0$ when $\omega=0$, we obtain

$$
\left\langle N_{\partial \Sigma}^{\Sigma}, N_{\partial \Sigma}^{\partial \Omega}\right\rangle=-\frac{\partial \omega}{\partial \eta}+r^{2} \bar{L}(\omega)+\bar{Q}^{2}(\omega) \quad \text { on } \partial \Sigma_{p, r, \omega},
$$

because $\partial_{\nu} \omega=\frac{\partial \omega}{\partial \eta}($ since $\mu=1)$.

\section{Proof of Theorem 1.2 and expansions of the isoperimetric profile $I_{\Omega}$}

P. Bérard and D. Meyer [1982, Appendix C] have shown by a localization argument that the isoperimetric profile of a compact Riemannian manifold asymptotically approaches that of $\mathbb{R}^{n+1}$. V. Bayle and C. Rosales [2005, Proposition 2.1] proved that the relative isoperimetric profile of a domain $\Omega$ of a Riemannian manifold behaves like the profile of the half space $\mathbb{R}_{+}^{n+1}$. Precisely, with

$$
\begin{gathered}
I(r):=I_{\Omega}\left(\left|r B_{+}^{n+1}\right|\right)=\min _{\substack{E \subset \Omega \\
|E|_{g}=\left|r B_{+}^{n+1}\right|}} \mathscr{P}_{g}(E, \Omega), \\
I_{+}(r):=I_{\mathbb{R}_{+}^{n+1}}\left(\left|r B_{+}^{n+1}\right|\right)=\mathscr{P}\left(r B^{n+1}, \mathbb{R}_{+}^{n+1}\right),
\end{gathered}
$$

they proved:

Proposition 4.1. For any $\varepsilon>0$, there exists $r_{0}(\varepsilon)>0$ such that

$$
(1-\varepsilon) I_{+}(r) \leq I(r) \leq(1+\varepsilon) I_{+}(r), \quad \text { whenever } r \leq r_{0} .
$$

Notice that from this upper bound, an isoperimetric region with small volume must touch the boundary (perpendicularly) because otherwise it would contradict 
Bérard and Meyer's lower bound. Moreover this upper bound will help after suitable scaling together with the Heintze-Karcher inequality to obtain a uniform bound for the mean curvature of the minimizing hypersurface trapping a small volume; see [Morgan and Johnson 2000, Section 2].

We start by proving the following regularity result which were obtained in [Morgan and Johnson 2000] and under weaker assumptions in [Nardulli 2006] in compact Riemannian manifolds.

Lemma 4.2. There exists $r_{0}>0$ such that if $r \in\left(0, r_{0}\right)$ and $E \subset \Omega$ is any set such that $\mathscr{P}_{g}(E, \Omega)=I(r)$, there exist $p \in \partial \Omega$ and $\omega^{p, r}: S_{+}^{n} \rightarrow \mathbb{R}$ such that

$$
\overline{\partial E \cap \Omega}=F^{p}\left(r\left(1+\omega^{p, r} \overline{S_{+}^{n}}\right)\right)
$$

with $\left\|\omega^{p, r}\right\|_{\mathscr{C}^{2, \alpha}\left(S_{+}^{n}\right)}+\left\|\omega^{p, r}\right\|_{\mathscr{C}^{1, \alpha}\left(\overline{S_{+}^{n}}\right)} \rightarrow 0$ as $r \rightarrow 0$.

Proof. Let $E_{j} \subset \Omega$ such that $\mathscr{P}_{g}\left(E_{j}, \Omega\right)=I\left(r_{j}\right), r_{j} \rightarrow 0$ as $j \rightarrow+\infty$. Define $\Omega_{j}:=\left(1 / r_{j}\right) \Omega$ and $E_{j}^{\prime}:=\left(1 / r_{j}\right) E_{j}$, so $\left|E_{j}^{\prime}\right|_{g_{j}}=\left|B_{+}^{n+1}\right|$ and $\mathscr{P}_{g_{j}}\left(E_{j}^{\prime}, \Omega_{j}\right)=$ $\left(1 / r_{j}^{n}\right) \mathscr{P}_{g}\left(E_{j}, \Omega\right) \leq c^{\prime} I_{+}(1)$.

Following [Morgan and Johnson 2000, Section 2] with the help of Proposition 4.1, we may assume that there exists a constant $R>0$ such that

$$
\operatorname{diam}_{g_{j}}\left(E_{j}^{\prime}\right) \leq R
$$

and since $\partial E_{j}^{\prime}$ intersects $\partial \Omega_{j}$, then

$$
\sup _{e \in \partial E_{j}} \operatorname{dist}_{g_{j}}\left(e, \partial \Omega_{j}\right) \leq \operatorname{diam}_{g_{j}}\left(E_{j}^{\prime}\right) \leq R .
$$

We can let $p_{j} \in \partial \Omega_{j}$ and $U_{j} \subset \mathbb{R}_{+}^{n+1}$ be such that $E_{j}^{\prime}=F_{j}\left(U_{j}\right)$, where the map $F_{j}: \gamma_{j} B_{+}^{n+1} \rightarrow \Omega_{j}$ is defined by $F_{j}(\cdot):=\left(1 / r_{j}\right) F^{p_{j}}\left(r_{j}(\cdot)\right)$ and $\gamma_{j} \rightarrow \infty$ as $j \rightarrow \infty$.

For $j$ fixed and sufficiently large, let $h_{j}:=\left(F_{j}\right)_{*}\left(g_{j}\right)$ be the metric induced by $F_{j}$ on $\mathbb{R}_{+}^{n+1}$. Then $U_{j}$ minimizes the perimeter $\mathscr{P}_{h_{j}}\left(\cdot, \gamma_{j} B_{+}^{n+1}\right)$ in $\left(\gamma_{j} B_{+}^{n+1}, h_{j}\right)$ among sets enclosing its volume $\left|U_{j}\right|_{h_{j}}=\left|B_{+}^{n+1}\right|$ and also intersects $\partial \mathbb{R}_{+}^{n+1}=$ $\mathbb{R}^{n} \times\{0\}$ perpendicularly.

Now since $h_{j}$ is converging to the Euclidean metric, we get $\operatorname{diam}\left(U_{j}\right) \leq c$ for every large $j$. So $\mathscr{P}_{h_{j}}\left(U_{j}, \mathbb{R}_{+}^{n+1}\right) \leq c$, which implies that $\mathscr{P}\left(U_{j}, \mathbb{R}_{+}^{n+1}\right) \leq c$. Hence by compactness there exists $U \subset \mathbb{R}_{+}^{n+1}$ such that $D 1_{U_{j}}{ }^{*} D 1_{U}$. Furthermore by the trace theorem,

$$
1_{U_{j}}\left|\mathbb{R}^{n} \times\{0\} \stackrel{L^{1}}{\rightarrow} 1_{U}\right|_{\mathbb{R}^{n} \times\{0\}} .
$$

Now to see that $U$ is a minimizer, we take $V \subset \mathbb{R}^{n+1}$ such that $|V|=\left|B_{+}^{n+1}\right|$ and define $c_{j} \rightarrow 1$ such that $c_{j}|V|_{h_{j}}=\left|B_{+}^{n+1}\right|$ (this is possible since $h_{j}$ too converges 
to the Euclidean metric). But then we have

$$
\mathscr{P}_{h_{j}}\left(U_{j}, \mathbb{R}_{+}^{n+1}\right) \leq c_{j}^{n /(n+1)} \mathscr{P}_{h_{j}}\left(V, \mathbb{R}_{+}^{n+1}\right)
$$

and this, together with the semicontinuity of the perimeter, implies that

$$
\mathscr{P}\left(U, \mathbb{R}_{+}^{n+1}\right) \leq \mathscr{P}\left(V, \mathbb{R}_{+}^{n+1}\right) .
$$

We conclude that $U$ is a minimizer in $\mathbb{R}_{+}^{n+1}$ among sets that enclose the volume $\left|B_{+}^{n+1}\right|$; therefore $U$ is a half-ball. Finally, again by results from [Morgan and Johnson 2000, Section 2], we have smooth convergence because mean curvatures are bounded. Hence we may assume that there exists $\omega^{p_{j}, r_{j}} \in \mathscr{C}^{2, \alpha}\left(S_{+}^{n}\right)$ such that

$$
\Sigma_{j}:=\partial U_{j} \cap \mathbb{R}_{+}^{n+1}=\left(1+\omega^{p_{j}, r_{j}}\right) S_{+}^{n}
$$

with $\left\|\omega^{p_{j}, r_{j}}\right\|_{\mathscr{C}^{2, \alpha}\left(S_{+}^{n}\right)}$ tending to zero as $j \rightarrow \infty$.

We now estimate the free boundary, $\mathscr{H}^{n}\left(\partial \Sigma_{j}\right)$, by slicing with hyperplanes $\mathbb{R}^{n} \times\{0\}+h N_{\partial \Omega}$ with $h \in \mathbb{R}$. For terminology, we refer the reader to [Morgan 1988]. In the following, with an abuse of notation, we will call $\Sigma_{j}$ the integer rectifiable current associated to the set $\Sigma_{j}$. We define $m_{j}(h)$ by

$$
m_{j}(h):=\mathscr{H}^{n}\left(\Sigma_{j} \cap\{d<h\}\right)=\mathscr{P}\left(U_{j},\left\{d_{j}<h\right\}\right) \text { for } 0<h<\frac{1}{2},
$$

where $\mathbb{R}^{n+1} \ni x \mapsto d(x)=x^{n+1}$ is the distance function from $\partial \mathbb{R}_{+}^{n+1}=\mathbb{R}^{n} \times\{0\}$. For $h \geq 0$ we consider the slice

$$
\left\langle\Sigma_{j}, d, h_{+}=\left(\partial \Sigma_{j}\right)\left\llcorner\{d>h\}-\partial\left(\Sigma_{j}\llcorner\{d>h\}) .\right.\right.\right.
$$

We deduce that $\left\langle\Sigma_{j}, d, 0_{+}\right\rangle=\partial \Sigma_{j}$. From [Morgan 1988, Section 4.11, (3)], we then get

$$
\mathscr{H}^{n-1}\left(\left\langle\Sigma_{j}, d, 0_{+}\right\rangle\right) \leq \operatorname{Lip}(d) \liminf _{h \searrow 0} \frac{m_{j}(h)}{h}=\liminf _{h \searrow 0} \frac{m_{j}(h)}{h},
$$

the equality being a consequence of $\operatorname{Lip}(d)=1$. Since $m_{j}(h)$ is increasing the same argument yields, for $\mathscr{L}^{1}$ a.e. $h>0$,

$$
\mathscr{H}^{n-1}\left(\left\langle\Sigma_{j}, d, h_{+}\right\rangle\right) \leq m_{j}^{\prime}(h) .
$$

By (10) and Lemma A.3,

$$
\begin{aligned}
m_{j}(h) & =\mathscr{P}\left(U_{j},\{d<h\}\right)=\mathscr{H}^{n}\left(\left(1+\omega^{p_{j}, r_{j}}\right) S_{+}^{n} \cap\{d<h\}\right) \\
& \leq h\left(1+O\left(r_{j}\right)\right) \mathscr{H}^{n-1}\left(\left\langle\Sigma_{j}, d, h_{+}\right\rangle\right) \leq h\left(1+O\left(r_{j}\right)\right) m_{j}^{\prime}(h) .
\end{aligned}
$$

Hence we get $m_{j}(h) \leq 2 h m_{j}^{\prime}(h)$, which is equivalent to

$$
\left(\frac{m_{j}(h)}{h}+2 m_{j}(h)\right)^{\prime} \geq 0
$$


for every $\mathscr{L}^{1}$ a.e. $\frac{1}{2}>h>0$. From this and the fact that $m_{j}$ is increasing we conclude that

$$
\mathscr{H}^{n-1}\left(\partial \Sigma_{j}\right) \leq \mathscr{H}^{n}\left(\Sigma_{j}\llcorner\{d<h\})\left(\frac{1}{h}+2\right) \quad \text { for every } h \in\left(0, \frac{1}{2}\right) .\right.
$$

From this together with Lemma A.3 we have

$$
\mathscr{H}^{n-1}\left(\partial \Sigma_{j}\right) \leq c \mathcal{H}^{n}\left(\Sigma_{j}\right) \leq \tilde{c} \text { for any large } j .
$$

Consequently, $\Sigma_{j}$ is an integral current, and by compactness [Morgan 1988, 5.5], $\partial \Sigma_{j}$ converges weakly to $\partial S_{+}^{n}$. Since mean curvatures of $\Sigma_{j}$ are bounded (see also [Morgan and Johnson 2000, (2.4)]), $C^{1, \alpha}$ convergence up to the free boundary follows by [Grüter and Jost 1986]. Hence finally we can assume that $\omega^{p_{j}, r_{j}} \in$ $\mathscr{C}^{1, \alpha}\left(\overline{S_{+}^{n}}\right)$ if $j$ is sufficiently large with

$$
\partial E_{j}^{\prime} \cap \bar{\Omega}_{j}=\frac{1}{r_{j}} F^{p_{j}}\left(r_{j}\left(1+\omega^{p_{j}, r_{j}}\right) \overline{S_{+}^{n}}\right),
$$

and $\left\|\omega^{p_{j}, r_{j}}\right\|_{\mathscr{C}^{2, \alpha}\left(S_{+}^{n}\right)}+\left\|\omega^{p_{j}, r_{j}}\right\|_{\mathscr{C}^{1, \alpha}\left(\overline{S_{+}^{n}}\right)} \rightarrow 0$ as $r \rightarrow 0$.

Remark 4.3. When applying the first compactness result, namely $D 1_{U_{j}}{ }^{*} D 1_{U}$, we also have (by Rellich's theorem) that

$$
1_{U_{j}} \stackrel{L^{1}}{\rightarrow} 1_{U} .
$$

Since $U=B_{+}^{n+1}$, by [Nardulli 2006], $\partial U_{j} \cap \mathbb{R}_{+}^{n+1}$ can be written as a normal graph over $S_{+}^{n}$ by a smooth function $\omega^{p_{j}, r_{j}}$ for which $\left\|\omega^{p_{j}, r_{j}}\right\|_{\mathscr{C}^{2, \alpha}\left(S_{+}^{n}\right)} \rightarrow 0$ as $r \rightarrow 0$.

Note also that $C^{k, \alpha}$ regularity and estimates of $\omega^{p_{j}, r_{j}}$ can be obtained by a bootstrap argument using Proposition 3.2, as in [Morgan and Johnson 2000; Nardulli 2006].

The following lemma shows the smoothness of the center of mass $c(r, p, \omega) \in$ $\partial \Omega$ of the hypersurface $\left.\Sigma_{p, r, \omega}:=F^{p}\left(r(1+\omega) \overline{S_{+}^{n}}\right)\right)$ as a function in $r, p$ and $\omega$. The proof can be obtained from [Nardulli 2007, Lemmas 1.3-1.4], with slight modifications.

Lemma 4.4. There exists a smooth map $c: \mathbb{R} \times \partial \boldsymbol{\Omega} \times \mathscr{b}^{2, \alpha}\left(\overline{S_{+}^{n}}\right) \rightarrow \partial \boldsymbol{\Omega}$ such that

$$
\int_{\Sigma_{p, r, \omega}}\left(F^{c}\right)^{-1}(z) d \operatorname{vol}_{\Sigma_{p, r, \omega}}=0 .
$$

Moreover there exists a smooth vector field $X_{p, r, \omega}$ on $T_{p} \partial \Omega$ such that

$$
X_{p, 0, \omega}=\frac{\int_{S_{+}^{n}}(1+\omega)^{n} \widetilde{\Theta} \sqrt{\|d \omega\|^{2}+(1+\omega)^{2}} d \sigma}{\int_{S_{+}^{n}}(1+\omega)^{n-1} \sqrt{\|d \omega\|^{2}+(1+\omega)^{2}} d \sigma} .
$$

and $c(r, p, \omega)=\exp _{p}^{\partial \Omega}\left(r X_{p, r, \omega}\right)$. (Here d $\omega$ is the differential of $\left.\omega.\right)$ 
According to Proposition 3.2, with $H(p, r, \omega)$ being the mean curvature of $\Sigma_{p, r, \omega}$, we define $T(p, r, \cdot): \mathscr{C}^{2, \alpha}\left(\overline{S_{+}^{n}}\right) \rightarrow \mathscr{C}^{0, \alpha}\left(\overline{S_{+}^{n}}\right)$

$$
\int_{S_{+}^{n}} T(p, r, \omega) \omega^{\prime} d \sigma:=\int_{S_{+}^{n}} r H(p, r, \omega) \omega^{\prime} d \sigma-\oint_{\partial S_{+}^{n}}\left\langle N_{\partial \Sigma_{p, r, \omega}}^{\partial \Omega}, N_{\partial \Sigma_{p, r, \omega}}^{\Sigma_{p, r, \omega}}\right\rangle \omega^{\prime} d s,
$$

for every $\omega^{\prime} \in L^{2}$.

Consider the eigenvalue problem

$$
\Delta_{S_{+}^{n}} u=\lambda u \quad \text { in } S_{+}^{n}, \quad \frac{\partial u}{\partial \eta}=0 \quad \text { on } \partial S_{+}^{n} .
$$

Letting

$$
0=\lambda_{0}<\lambda_{1} \leq \lambda_{2} \leq \cdots \rightarrow \infty
$$

be the eigenvalues, up to a reflection, we know that $\lambda_{k}=n+k-1$ and the eigenspaces corresponding to $\lambda_{0}=0$ and $\lambda_{1}=n$ are

$$
\Lambda_{0}:=\operatorname{span}\{1\}, \quad \Lambda_{1}:=\operatorname{span}\left\{\Theta^{1}, \ldots, \Theta^{n}\right\} .
$$

We denote by $\Pi_{0}$ and $\Pi_{1}$ the $L^{2}$ projections onto these spaces and we define

$$
\Pi:=\mathrm{Id}-\Pi_{1}-\Pi_{0}, \quad \Pi_{1}^{\perp}:=\Pi_{0}+\Pi .
$$

We recall that $E_{p, r, \omega}$ is the set bounded by the hypersurface $\Sigma_{p, r, \omega}$ and $\partial \Omega$.

Definition 4.5. A set $E_{p, r, \omega}$ is called a pseudo-half-ball if $\Pi \circ T(p, r, \omega) \equiv 0$, or equivalently

$$
\Pi_{1}^{\perp} \circ T(p, r, \omega) \equiv \text { Const } \in \mathbb{R},
$$

where $T(p, r, \omega)$ is defined in (11).

Letting $\Xi \in T_{p} \partial \Omega$ be such that $\Pi_{1} \omega=\langle\Xi, \widetilde{\Theta}\rangle$, we get by Lemma 4.4

$$
c(r, p, \omega)=p+\frac{\left|S_{+}^{n}\right|}{n+1} r \Xi+r^{2}\left\{L_{p}(\omega)+\mathcal{O}(r)+Q_{p}(\omega)\right\}^{\alpha} E_{\alpha} .
$$

From the expansion of the volume of the sets $E_{p, r, \omega}$ (Lemma A.4), we define $\Phi(p, r, \omega):=r^{-n-1}\left|E_{p, r, \omega}\right|_{g}-\left|B_{+}^{n+1}\right|=\int_{S_{+}^{n}} \omega d \sigma+\mathcal{O}(r)+\int_{S_{+}^{n}}\left(\mathcal{O}(r) \omega+\hat{Q}_{p}(\omega)\right) d \sigma$.

It turns out that

$$
\Phi(p, 0,0)=0, \quad \frac{\partial \Phi(p, 0,0)}{\partial \omega}[u]=\Pi_{0} u .
$$

Now, we can associate to any hypersurface $\Sigma_{p, r, \omega}$ the smooth mapping

$$
\begin{gathered}
\Psi: \partial \Omega \times(0,1) \times \mathscr{C}^{2, \alpha}\left(\overline{S_{+}^{n}}\right) \rightarrow T_{p} \partial \Omega \times \Pi \mathscr{C}^{0, \alpha}\left(\overline{S_{+}^{n}}\right) \times \mathbb{R}, \\
\Psi(p, r, \omega):=\left(\frac{n+1}{\left|S_{+}^{n}\right|} X_{p, r, \omega}, \Pi \circ T(p, r, \omega),-n \Phi(p, r, \omega)\right) .
\end{gathered}
$$


Lemma 4.6. There exist $r_{0}>0$ and $c_{0}>0$ such that for any $p \in \partial \Omega$ and $r \in\left(0, r_{0}\right)$, there exists a unique smooth $\omega^{p, r} \in \mathscr{C}^{2, \alpha}\left(\overline{S_{+}^{n}}\right)$ with

$$
\left\|\omega^{p, r}\right\|_{\mathscr{C}^{2, \alpha}\left(\overline{S_{+}^{n}}\right)} \leq c_{0} r_{0}
$$

such that $\Psi\left(p, r, \omega^{p, r}\right)=(0,0,0)$, namely

$$
c\left(r, p, \omega^{p, r}\right)=p, \quad \Pi_{1}{ }^{\perp} \circ T\left(p, r, \omega^{p, r}\right) \in \mathbb{R} \quad \text { and } \quad\left|E_{p, r, \omega^{p, r}}\right|_{g}=\left|r B_{+}^{n+1}\right|
$$

for every $r \in\left(0, r_{0}\right)$.

Proof. We make the identification $\mathscr{G}^{k, \alpha}\left(\overline{S_{+}^{n}}\right) \equiv T_{p} \partial \Omega \times \Pi \mathscr{C}^{k, \alpha}\left(\overline{S_{+}^{n}}\right) \times \mathbb{R}$. Any $u \in$ $\mathscr{C}^{k, \alpha}\left(\overline{S_{+}^{n}}\right)$ has a decomposition $u=\langle\Xi, \widetilde{\Theta}\rangle+\Pi u+u_{0}=\langle\Xi, \widetilde{\Theta}\rangle+w$.

It is easy to see that $\Psi(p, 0,0)=(0,0,0)$ while

$$
\left\langle\frac{\partial \Psi}{\partial \omega}(p, 0,0)[u], u^{\prime}\right\rangle=\int_{S_{+}^{n}} \nabla_{S_{+}^{n}} w \nabla_{S_{+}^{n}} w^{\prime}-n w w^{\prime} d \sigma+\int_{S_{+}^{n}}\langle\Xi, \widetilde{\Theta}\rangle\left\langle\Xi^{\prime}, \widetilde{\Theta}\right\rangle d \sigma .
$$

Since $\frac{\partial \Psi}{\partial \omega}(p, 0,0)$ is an isomorphism from $\mathscr{C}^{2, \alpha}\left(\overline{S_{+}^{n}}\right)$ in to $\mathscr{C}^{0, \alpha}\left(\overline{S_{+}^{n}}\right)$, the lemma follows by the implicit function theorem.

By choosing $r_{0}$ small enough in Lemma 4.6, we may assume that the hypersurfaces $\Sigma_{p, r, \omega^{p, r}}$ are embedded in $\Omega$ for any $r \in\left(0, r_{0}\right)$, since $\left\|\omega^{p, r}\right\|_{\mathscr{C}_{1, \alpha}\left(\overline{S_{+}^{n}}\right)} \rightarrow 0$ as $r_{0} \rightarrow 0$. For simplicity, we will call $E_{p, r}:=E_{p, r, \omega^{p, r}}$ the sets bounded by $\Sigma_{p, r, \omega^{p, r}}$ and $\partial \Omega$.

The preceding lemma yields, for any fixed $r \in\left(0, r_{0}\right)$, a manifold of pseudohalf-balls diffeomorphic to $\partial \Omega$ and having volume $\left|r B_{+}^{n+1}\right|$ equal to

$\mathfrak{C}_{r}:=\left\{E_{p, r, \omega^{p, r}} \subset \Omega: \Psi\left(p, r, \omega^{p, r}\right)=(0,0,0),\left\|\omega^{p, r}\right\|_{\mathscr{C}^{2, \alpha}\left(\overline{S_{+}^{n}}\right)} \leq c_{0} r_{0}, p \in \partial \Omega\right\}$.

Lemma 4.7. If $r \ll 1$, then

$$
I(r)=\inf _{E \in \mathfrak{C}_{r}} \mathscr{P}_{g}(E, \Omega)=\inf _{p \in \partial \Omega} \mathscr{P}_{g}\left(E_{p, r}, \Omega\right),
$$

where $E_{p, r}, p \in \partial \Omega$, ranges over the elements of $\mathfrak{C}_{r}$.

Proof. Let $E$ be a solution to the isoperimetric problem with $|E|_{g}=\left|r B_{+}^{n+1}\right|$. We have to check that $E$ belongs to $\mathfrak{C}_{r}$ if $r$ is small enough. Assume that $r \ll 1$. Lemma 4.2 implies that $\overline{\partial E \cap \Omega}=F^{q}\left(r\left(1+u^{q, r}\right) \overline{S_{+}^{n}}\right)$ for some $q \in \partial \Omega$ and $\left\|u^{q, r}\right\|_{\mathscr{C}^{2, \alpha}\left(\overline{S_{+}^{n}}\right)}$ approaching 0 as $r \rightarrow 0$.

Let $p \in \partial \Omega$ be the center of mass of $\partial E$. By (13),

$$
\operatorname{dist}_{g}(p, q) \leq c\left(r^{2}+r\left\|u^{q, r}\right\|_{\mathscr{C}^{2, \alpha}\left(\overline{S_{+}^{n}}\right)}\right)
$$

so if $r \ll 1$, we can find $v(p, r)$ with $\|v(p, r)\|_{\mathscr{C}^{2, \alpha}\left(\overline{S_{+}^{n}}\right)} \rightarrow 0$ as $r \rightarrow 0$ such that

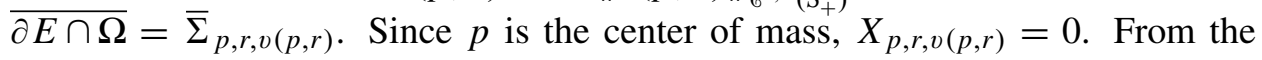
mean curvature expansions, we get $\Pi \circ T(p, r, v(p, r))=0$ because the mean 
curvature of $\partial E$ is constant and $\partial E$ intersects $\partial \Omega$ perpendicularly. Consequently $\Psi(p, r, v(p, r))=(0,0,0)$. We conclude that $E \in \mathfrak{C}_{r}$ if $r$ is small enough.

Lemma 4.8. For any $E_{p, r} \in \mathfrak{C}_{r}$, we have

$$
\begin{aligned}
& \mathscr{P}_{g}\left(E_{p, r}, \Omega\right) \\
& \quad=\mathscr{P}\left(r B^{n+1}, \mathbb{R}_{+}^{n+1}\right)-\frac{n}{n+2} \frac{\left|B^{n}\right|}{\left|B_{+}^{n+1}\right|} H_{\partial \Omega}(p)\left|r B_{+}^{n+1}\right|+O_{p}\left(\left|r B_{+}^{n+1}\right|^{(n+2) /(n+1)}\right),
\end{aligned}
$$

where $O_{p}(\rho)$ is smooth and tends to zero as $\rho \rightarrow 0$ uniformly in $p$.

Proof. Let $E_{p, r} \in \mathfrak{C}_{r}$. Differentiating $\Phi\left(p, r, \omega^{p, r}\right)=0$ with respect to $r$, we deduce

$$
\Pi_{0} \omega^{p, r}=\int_{S_{+}^{n}} \omega^{p, r} d \sigma=-\frac{r}{n+2}\left\langle S_{p}\left(E_{i}\right), E_{i}\right\rangle \int_{S_{+}^{n}} \Theta^{n+1} d \sigma+O_{p}\left(r^{2}\right) .
$$

Together with Lemma A.3, this gives

$r^{-n} \mathscr{P}_{g}\left(E_{p, r}, \Omega\right)$

$=\mathscr{P}\left(B^{n+1}, \mathbb{R}_{+}^{n+1}\right)+r \int_{S_{+}^{n}}\left(\left\langle S_{p}\left(E_{i}\right), E_{i}\right\rangle-\left\langle S_{p}(\widetilde{\Theta}), \widetilde{\Theta}\right\rangle\right) \Theta^{n+1} d \sigma+n r \int_{S_{+}^{n}} \omega_{0}^{p} d \sigma+O_{p}\left(r^{2}\right)$

$=\mathscr{P}\left(B^{n+1}, \mathbb{R}_{+}^{n+1}\right)+\frac{2 r}{n+2}\left\langle S_{p}\left(E_{i}\right), E_{i}\right\rangle \int_{S_{+}^{n}} \Theta^{n+1} d \sigma-\int_{S_{+}^{n}}\left\langle S_{p}(\widetilde{\Theta}), \widetilde{\Theta}\right\rangle \Theta^{n+1} d \sigma+O_{p}\left(r^{2}\right)$.

Recall that $H_{\partial \Omega}(p)=-\frac{1}{n}\left\langle S_{p}\left(E_{i}\right), E_{i}\right\rangle$. Moreover since

$$
\int_{S_{+}^{n}}\left\langle S_{p}(\widetilde{\Theta}), \widetilde{\Theta}\right\rangle \Theta^{n+1} d \sigma=\left\langle S_{p}\left(E_{i}\right), E_{j}\right\rangle \int_{S_{+}^{n}} \Theta^{i} \Theta^{j} \Theta^{n+1} d \sigma
$$

and observing that $\int_{S_{+}^{n}} \Theta^{i} \Theta^{j} \Theta^{n+1} d \sigma=0$ if $i \neq j$, we deduce that

$$
\mathscr{P}_{g}\left(E_{p, r}, \Omega\right)=\mathscr{P}\left(r B^{n+1}, \mathbb{R}_{+}^{n+1}\right)-c_{n} H_{\partial \Omega}(p)\left|r B_{+}^{n+1}\right|+O_{p}\left(\left|r B_{+}^{n+1}\right|^{(n+2) /(n+1)}\right),
$$
with

$$
c_{n}=\frac{n}{\left|B_{+}^{n+1}\right|} \int_{S_{+}^{n}}\left(\frac{2}{n+2}-\left(\Theta^{1}\right)^{2}\right) \Theta^{n+1} d \sigma=\frac{n}{n+2} \frac{\left|B^{n}\right|}{\left|B_{+}^{n+1}\right|} .
$$

We have used equalities

$$
\int_{S_{+}^{n}} \Theta^{n+1} d \sigma=\frac{\operatorname{Area} S^{n-1}}{n}=\left|B^{n}\right|, \quad \int_{S_{+}^{n}}\left(\Theta^{i}\right)^{2} \Theta^{n+1} d \sigma=\frac{\operatorname{Area} S^{n-1}}{n(n+2)} .
$$

The proof of Theorem 1.2 is finalized by the following:

Lemma 4.9. Let $r_{k}$ be a sequence tending to 0 and let $E_{k} \subset \Omega$ satisfy $\left|E_{k}\right|_{g}=$ $\left|r_{k} B_{+}^{n+1}\right|$ and $\mathscr{P}_{g}\left(E_{k}, \Omega\right)=I\left(r_{k}\right)$. Let $p_{k} \in \partial \Omega$ be the center of mass of $\partial E_{k}$, and suppose $p_{k}$ converges to a point $p \in \partial \Omega$. Then

$$
H_{\partial \Omega}(p)=\max _{q \in \partial \Omega} H_{\partial \Omega}(q) .
$$


Proof. If $k$ is large enough, $E_{k}=E_{p_{k}, r_{k}} \in \mathfrak{C}_{r_{k}}$ and also by Lemma 4.7 we have that

$$
\mathscr{P}_{g}\left(E_{p_{k}, r_{k}}, \Omega\right)=I\left(r_{k}\right)=\min _{q \in \partial \Omega} \mathscr{P}_{g}\left(E_{q, r_{k}}, \Omega\right)
$$

where $E_{p, r_{k}}, p \in \partial \Omega$, denote the elements of $\mathfrak{C}_{r_{k}}$. Now by Lemma 4.8, we have

$$
-H_{\partial \Omega}\left(p_{k}\right)+O\left(p_{k}, r_{k}\right)=\min _{q \in \partial \Omega}\left(-H_{\partial \Omega}(q)+O\left(q, r_{k}\right)\right)
$$

with $\left|O\left(p_{k}, r_{k}\right)\right| \rightarrow 0$ and $\sup _{q \in \partial \Omega}\left|O\left(q, r_{k}\right)\right| \rightarrow 0$ when $k$ tends to infinity. The lemma then follows by taking $k$ to infinity.

\section{Appendix A: Expansions for the area and enclosed volume}

Consider a hypersurface $\partial E_{p, r, \omega}=\Sigma_{p, r, \omega}$. Recall that the mapping

$$
z \mapsto G(z)=F^{p}\left(r(1+\omega) \widetilde{\Theta}(z), r(1+\omega) \Theta^{n+1}(z)\right)
$$

(see (4) and (5)) parametrizes $\Sigma_{p, r, \omega}$ and its tangent space is spanned by the vector fields

$$
Z_{j}=G_{*}\left(\partial_{z^{j}}\right)=r(1+\omega) \Upsilon_{j}+r \omega_{j} \Upsilon, \quad j=1, \ldots, n .
$$

At the point $G(z)$, Proposition 2.2 yields

$$
\begin{aligned}
\left\langle X_{i}, X_{j}\right\rangle= & \delta_{i j}+2 r(1+\omega)\left\langle S\left(\tilde{\Upsilon}_{i}\right), \tilde{\Upsilon}_{j}\right\rangle \Theta^{n+1}+\frac{1}{3} r^{2}(1+\omega)^{2}\left\langle R_{p}\left(\widetilde{\Theta}, \widetilde{\Theta}_{i}\right) \widetilde{\Theta}_{,} \widetilde{\Theta}_{j}\right\rangle \\
& +\left(\left\langle R_{p}^{\mu}\left(N_{\partial \Omega}, \widetilde{\Theta}_{i}\right) N_{\partial \Omega}, \widetilde{\Theta}_{j}\right\rangle+\left\langle S\left(\tilde{\Upsilon}_{i}\right), S\left(\tilde{\Upsilon}_{j}\right)\right\rangle\right)\left(\Theta^{n+1}\right)^{2} \\
& +\mathcal{O}\left(r^{3}\right)+r^{3} L(\omega)+r^{3} Q(\omega), \\
\left\langle X_{i},\right. & \left.X_{n+1}\right\rangle=\mathcal{O}\left(r^{3}\right)+r^{3} L(\omega)+r^{3} Q(\omega), \quad\left\langle X_{n+1}, X_{n+1}\right\rangle=1 .
\end{aligned}
$$

Letting $g_{i j}^{\Sigma_{p, r, \omega}}:=\left\langle Z_{i}, Z_{j}\right\rangle$, we then get the first fundamental form of $\Sigma_{p, r, \omega}$.

\section{Proposition A.1.}

$$
\begin{aligned}
(1+ & \omega)^{-2} r^{-2} g_{i j}^{\Sigma_{p, r, \omega}} \\
= & \mu^{2} \delta_{i j}+\left(2 \omega_{j}\left\langle S(\widetilde{\Upsilon}), \widetilde{\Upsilon}_{i}\right\rangle+2 \omega_{i}\left\langle S(\widetilde{\Upsilon}), \widetilde{\Upsilon}_{j}\right\rangle+2(1+\omega)\left\langle S\left(\widetilde{\Upsilon}_{j}\right), \widetilde{\Upsilon}_{i}\right\rangle\right) r \Theta^{n+1} \\
& +\omega_{i} \omega_{j}+\frac{1}{3}\left\langle R_{p}\left(\widetilde{\Theta}, \widetilde{\Theta}_{i}\right) \widetilde{\Theta}, \widetilde{\Theta}_{j}\right\rangle r^{2}+\left\langle S\left(\widetilde{\Upsilon}_{j}\right), S\left(\widetilde{\Upsilon}_{i}\right)\right\rangle r^{2}\left(\Theta^{n+1}\right)^{2} \\
& +\left(\Theta^{n+1}\right)^{2}\left\langle R_{p}^{\mu}\left(N_{\partial \Omega}, \widetilde{\Theta}_{i}\right) N_{\partial \Omega}, \widetilde{\Theta}_{i}\right\rangle r^{2}+\mathcal{O}\left(r^{3}\right)+r^{2} L(\omega)+r Q^{2}(\omega)+Q^{3}(\omega) .
\end{aligned}
$$

Using this formula we can deduce the expansion of the volume form. 
Lemma A.2. Under the notation above, the volume form expands as

$$
\begin{aligned}
& r^{n} \sqrt{\operatorname{det} g^{\Sigma_{p, r, \omega}}}= \\
& \mu^{n}+r \Theta^{n+1} \mu^{n-2}\left\langle S\left(\tilde{\Upsilon}_{i}\right), \tilde{\Upsilon}_{i}\right\rangle+n \omega \mu^{n} \\
& +r \Theta^{n+1} \mu^{n-2}\left(3(n+1) \omega\left\langle S\left(\tilde{\Upsilon}_{i}\right), \tilde{\Upsilon}_{i}\right\rangle+2 \omega_{i}\langle\tilde{\Upsilon}, \tilde{\Upsilon}\rangle\right) \\
& +\frac{1}{2} r^{2}\left(\Theta^{n+1}\right)^{2} \mu^{n-2}\left(\left\langle S\left(\tilde{\Upsilon}_{i}\right), S\left(\tilde{\Upsilon}_{i}\right)\right\rangle+\mu^{-2}\left|\left\langle S\left(\tilde{\Upsilon}_{i}\right), \tilde{\Upsilon}_{i}\right\rangle\right|^{2}-2 \mu^{-2}\left|\left\langle S\left(\tilde{\Upsilon}_{i}\right), \tilde{\Upsilon}_{j}\right\rangle\right|^{2}\right) \\
& +\frac{1}{6} r^{2} \mu^{n-2}\left\langle R_{p}\left(\widetilde{\Theta}, \widetilde{\Theta}_{i}\right) \widetilde{\Theta}, \widetilde{\Theta}_{i}\right\rangle+\frac{1}{2} r^{2}\left(\Theta^{n+1}\right)^{2} \mu^{n-2}\left\langle R_{p}^{\mu}\left(N_{\partial \Omega}, \widetilde{\Theta}_{i}\right) N_{\partial \Omega}, \widetilde{\Theta}_{i}\right\rangle \\
& +\frac{1}{2} \mu^{n-2}\left(\omega_{i}^{2}+n(n-1) \mu^{2} \omega^{2}\right)+\mathcal{O}\left(r^{3}\right)+r^{2} L(\omega)+r Q^{2}(\omega)+Q^{3}(\omega) \text {. }
\end{aligned}
$$

Observe that

$$
\begin{aligned}
& \left.\left\langle S\left(Y_{k}\right), Y_{l}\right\rangle\right|_{f^{p}(r(1+\omega) \widetilde{\Theta})} \\
& \quad=\left\langle S\left(E_{k}\right), E_{l}\right\rangle+\left\langle T\left(\widetilde{\Theta}, E_{k}\right), E_{l}\right\rangle+\mathcal{O}\left(r^{2}\right)+r L(\omega)+Q(\omega)
\end{aligned}
$$

where $T\left(Y_{i}, Y_{k}\right)=\nabla_{Y_{i}} \nabla_{Y_{k}} N_{\partial \Omega}$. In fact we have

$$
Y_{i}\left\langle S\left(Y_{k}\right), Y_{l}\right\rangle=\left\langle T\left(Y_{i}, Y_{k}\right), Y_{l}\right\rangle+\left\langle\nabla_{Y_{k}} N_{\partial \Omega}, \nabla_{Y_{i}} Y_{l}\right\rangle
$$

By the parallel transport of the vector fields $Y_{j}$ with respect to the connection $\nabla^{\partial \Omega}$ of $\partial \Omega$, we have $\left.\nabla_{Y_{i}}^{\partial \Omega} Y_{l}\right|_{p}=0$. Since $\nabla_{Y_{i}} Y_{l}=\nabla_{Y_{i}}^{\partial \Omega} Y_{l}-\left\langle S\left(Y_{i}\right), Y_{l}\right\rangle N_{\partial \Omega}$, it follows that $\left.\left\langle\nabla_{Y_{k}} N_{\partial \Omega}, \nabla_{Y_{i}} Y_{l}\right\rangle\right|_{p}=0$.

Lemma A.3. The area of the hypersurface $\Sigma_{p, r, \omega}$ has the following expansion:

$$
\begin{aligned}
& r^{-n} A\left(\Sigma_{p, r, \omega}\right) \\
& =\mathscr{P}\left(B^{n+1}, \mathbb{R}_{+}^{n+1}\right)+r \int_{S_{+}^{n}}\left(\left\langle S\left(E_{i}\right), E_{i}\right\rangle-\langle S(\widetilde{\Theta}), \widetilde{\Theta}\rangle\right) \Theta^{n+1} d \sigma+n \int_{S_{+}^{n}} \omega d \sigma \\
& \quad+3 r(n+1) \int_{S_{+}^{n}}\left(\left\langle S\left(E_{i}\right), E_{i}\right\rangle-\langle S(\widetilde{\Theta}), \widetilde{\Theta}\rangle\right) \Theta^{n+1} \omega d \sigma \\
& \left.\quad+2 r \int_{S_{+}^{n}} \Theta^{n+1}\left\langle S(\widetilde{\Theta}), \nabla_{S^{n}} \omega\right)\right\rangle d \sigma \\
& \quad+\frac{1}{2} r^{2} \int_{S_{+}^{n}}\left(\left(\left\langle S\left(E_{i}\right), S\left(E_{i}\right)\right\rangle-\langle S(\widetilde{\Theta}), S(\widetilde{\Theta})\rangle\right)\right. \\
& \left.\quad+\left(\left\langle S\left(E_{i}\right), E_{i}\right\rangle-\langle S(\widetilde{\Theta}), \widetilde{\Theta}\rangle\right)^{2}\right)\left(\Theta^{n+1}\right)^{2} d \sigma \\
& \quad-\frac{1}{2} r^{2} \int_{S_{+}^{n}}\left(|\langle S(\widetilde{\Theta}), \widetilde{\Theta}\rangle|^{2}-2\left|\left\langle S\left(E_{i}\right), E_{i}\right\rangle\right|^{2}+\left|\left\langle S\left(E_{j}\right), S\left(E_{i}\right)\right\rangle\right|^{2}\right) d \sigma \\
& \left.\left.\left.\quad-\frac{1}{6} r^{2} \int_{S_{+}^{n}} \operatorname{Ric}_{p}(\widetilde{\Theta}, \widetilde{\Theta}) d \sigma \quad \widetilde{\Theta}\right) N_{\partial \Omega}, \widetilde{\Theta}\right\rangle\right)\left(\Theta^{n+1}\right)^{2} d \sigma \\
& \quad-\frac{1}{2} r^{2} \int_{S_{+}^{n}}\left(\operatorname{Ric}_{p}^{\mathcal{M}}(\widetilde{\Theta}, \widetilde{\Theta})+\left\langle R_{p}^{\mu}\left(N_{\partial \Omega}, \widetilde{\Theta}\right)\right.\right. \\
& \quad+\frac{1}{2} \int_{S_{+}^{n}}\left(\left|\nabla_{S^{n}} \omega\right|^{2}+n(n-1) \omega^{2}\right) d \sigma+O_{p}\left(r^{3}\right)+\int_{S_{+}^{n}}\left(r^{2} L(\omega)+r Q^{2}(\omega)+Q^{3}(\omega)\right) d \sigma .
\end{aligned}
$$


Use Proposition 2.2 and (17) to have the volume form of $B^{\mathcal{M}}(p, \rho)$ in $\mathcal{M}$ for $\rho$ small:

$$
\begin{aligned}
\rho^{-n} \sqrt{\operatorname{det} g_{i j}}=1 & +\rho \Theta^{n+1}\left(\left\langle S\left(E_{i}\right), E_{i}\right\rangle+\rho\left\langle T\left(\widetilde{\Theta}, E_{i}\right), E_{i}\right\rangle\right) \\
& +\frac{1}{2} \rho^{2}\left\langle R_{p}^{\mu}\left(N_{\partial \Omega}, E_{i}\right) N_{\partial \Omega}, E_{i}\right\rangle+\frac{1}{6} \rho^{2}\left\langle R_{p}\left(\widetilde{\Theta}, E_{i}\right) \widetilde{\Theta}, E_{i}\right\rangle \\
& +\rho^{2}\left(\Theta^{n+1}\right)^{2}\left\langle R_{p}^{\mu}\left(N_{\partial \Omega}, E_{i}\right) N_{\partial \Omega}, E_{i}\right\rangle+\frac{1}{2} \rho^{2}\left(\Theta^{n+1}\right)^{2}\left\langle S\left(E_{i}\right), S\left(E_{i}\right)\right\rangle \\
& +\frac{1}{8} \rho^{2}\left(\Theta^{n+1}\right)^{2}\left|\left\langle S\left(E_{i}\right), E_{j}\right\rangle\right|^{2}-\frac{1}{4} \rho^{2}\left(\Theta^{n+1}\right)^{2}\left|\left\langle S\left(E_{i}\right), E_{k}\right\rangle\right|^{2}+\mathcal{O}\left(\rho^{3}\right) .
\end{aligned}
$$

Integration over the set $\rho \leq r(1+\omega)$ gives the expansion of the volume bounded by $\Sigma_{p, \omega, r}$ and $\partial \Omega$.

Lemma A.4. The following expansion holds:

$$
\begin{aligned}
r^{-n-1}|E(p, r, \omega)|_{g} \\
=\frac{1}{n+1} \mathscr{P}\left(B^{n+1}, \mathbb{R}_{+}^{n+1}\right)+\frac{r}{n+2}\left\langle S\left(E_{i}\right), E_{i}\right\rangle \int_{S_{+}^{n}} \Theta^{n+1} d \sigma+\int_{S_{+}^{n}} \omega d \sigma \\
\quad+\frac{r^{2}}{n+3}\left(\frac{1}{8}\left|\left\langle S\left(E_{i}\right), E_{i}\right\rangle\right|^{2}+\frac{1}{2}\left\langle S\left(E_{i}\right), S\left(E_{i}\right)\right\rangle-\frac{1}{4}\left|\left\langle S\left(E_{i}\right), E_{j}\right\rangle\right|^{2}\right) \int_{S_{+}^{n}}\left(\Theta^{n+1}\right)^{2} d \sigma \\
\quad+-\frac{r^{2}}{6(n+3)} \int_{S_{+}^{n}} \operatorname{Ric}_{p}(\widetilde{\Theta}, \widetilde{\Theta}) d \sigma-\frac{r^{2}}{2(n+3)} \operatorname{Ric}_{p}^{\mu}\left(N_{\partial \Omega}, N_{\partial \Omega}\right) \int_{S_{+}^{n}}\left(\Theta^{n+1}\right)^{2} d \sigma \\
\quad+r\left\langle S\left(E_{i}\right), E_{i}\right\rangle \int_{S_{+}^{n}} \Theta^{n+1} \omega d \sigma+\frac{n}{2} \int_{S_{+}^{n}} \omega^{2} d \sigma+O_{p}\left(r^{3}\right) \\
\quad+\int_{S_{+}^{n}}\left(\mathcal{O}\left(r^{2}\right) \omega+\mathcal{O}(r) \hat{Q}^{2}(\omega)+\hat{Q}^{3}(\omega)\right) d \sigma,
\end{aligned}
$$

where $\hat{Q}^{a}(\omega)$ is a polynomial in $w$ of degree at least a, with smooth coefficients depending on $p, \Theta$ and maybe on $r$ but uniformly bounded by a constant depending only on $\Omega$.

\section{Appendix B: Existence of orthogonally intersecting hemispheres of constant mean curvature centered on $\partial B$}

Let $E$ be an open smooth subset of $\Omega$ and $\Sigma:=\partial E \cap \Omega$. Assume that the boundary of $\partial \Sigma$ is nonempty and is contained in $\partial \Omega$. From the first variation of area (see for instance [Ros and Vergasta 1995]), $E$ is a critical point for the perimeter functional under variations that keep the volume invariant if and only if

$$
n H_{\Sigma} \equiv \text { const in } \Sigma \quad \text { and }\left\langle N_{\partial \Sigma}^{\Sigma}, N_{\partial \Sigma}^{\partial \Omega}\right\rangle_{g}=0 \text { in } \partial \Sigma,
$$

where for $B \subset A$, the expression $N_{B}^{A}$ denotes the unit outer normal of $B$ in $A$ while $H_{\Sigma}$ is the mean curvature of $\Sigma$. 
We have seen in Section 4 that solutions $E_{r}$ to the isoperimetric problem trapping a volume $\left|r B_{+}^{n+1}\right|$ have mean curvatures $H_{\partial E_{r}}$ blowing up and in fact $H_{\partial E_{r}} \sim n / r$. Moreover their boundaries are normal graphs over a hemisphere centered at some point in $\partial \Omega$. It is therefore natural to study the existence of disk-type solutions to the problem

$$
H_{\Sigma} \equiv \frac{n}{r} \text { in } \Sigma, \quad \partial \Sigma \subset \partial \Omega \text { and }\left\langle N_{\partial \Sigma}^{\Sigma}, N_{\partial \Sigma}^{\partial \Omega}\right\rangle_{g}=0 \text { on } \partial \Sigma .
$$

Proposition B.1. There exist $r_{0}>0$ and a smooth function $f:\left(0, r_{0}\right) \times \partial \Omega \rightarrow \mathbb{R}$ such that for every $r \in\left(0, r_{0}\right)$, if $p$ is a critical point of $f(r, \cdot)$ then (18) admits a solution $\Sigma_{p, r}$ which is a normal graph over $F^{p}\left(r S_{+}^{n}\right)$. Furthermore

$$
\left\|f(r, \cdot)-H_{\partial \Omega}\right\|_{\mathscr{C}^{1}(\partial \Omega)} \leq c r
$$

for some positive constant $c$.

Let us describe the proof of this. We have to recall that we look for stationary sets with a given profile for the total energy functional

$$
\mathscr{E}_{r}(E)=\mathscr{P}_{g}(E, \Omega)+\frac{n}{r}|E|_{g} .
$$

The set $Z_{r}:=\left\{F^{p}\left(r B_{+}^{n+1}\right), p \in \partial \Omega\right\}$ is a manifold of approximate solutions for $\mathscr{E}_{r}$. Indeed, by Lemma A.3 and Lemma A.4,

$$
\mathscr{E}_{r}\left(F^{p}\left(r B_{+}^{n+1}\right)\right)=\mathcal{O}(r) .
$$

Due to the invariance by translations when $\partial \Omega=\mathbb{R}^{n}$ is "flat", the linearized mean curvature operator together with the orthogonality conditions (see Proposition 3.2) may have small (possibly zero) eigenvalues, so we cannot invert it to apply a fixed point argument to solve the problem. However we will perturb $Z_{r}$ to a manifold $\tilde{Z}_{r}$ of critical points for $\mathscr{E}$ modulo $n$ Lagrange-multipliers. The second step is to show that in fact $\tilde{Z}_{r}$ is a natural constraint for $\mathscr{E}$, namely a critical point of $\mathscr{E}^{\mathscr{E}} \tilde{Z}_{\tilde{Z}_{r}}$ is also stationary for $\mathscr{E}$. For that we use an argument of Kapouleas [1991] which was successfully employed in [Pacard and Xu 2009] to obtain constant mean curvature spheres in Riemannian manifolds.

This method is also closely related to variational-perturbative methods introduced by Ambrosetti and Badiale [1998] and subsequently used with success to get existence and multiplicity results for a wide class of variational problems in some perturbative settings. We refer to [Ambrosetti and Malchiodi 2006] for more details and related applications.

At this point, the reader may wish to review Proposition 3.2.

Define

$$
\left\langle\mathscr{L}_{0}(u), u^{\prime}\right\rangle:=\int_{S_{+}^{n}}\left(\nabla_{S_{+}^{n}} u \nabla_{S_{+}^{n}} u^{\prime}-n u u^{\prime}\right) d \sigma .
$$


Since the kernel of this operator is $\Lambda_{1}$ (see (12)), by the Fredholm theorem there exists a unique $\bar{\omega}^{p} \in \mathscr{C}^{2, \alpha}\left(S_{+}^{n}\right)$ such that

$$
\begin{cases}\mathscr{L}_{0} \bar{\omega}=\Theta^{n+1}\left((n+3)\langle S(\tilde{\Theta}), \tilde{\Theta}\rangle-\left\langle S\left(E_{i}\right), E_{i}\right\rangle\right) & \text { in } S_{+}^{n}, \\ \frac{\partial \bar{\omega}}{\partial \eta}=0 & \text { on } \partial S_{+}^{n},\end{cases}
$$

because of the evenness of the right hand side. Moreover $\bar{\omega}^{p}$ satisfies

$$
n \int_{S_{+}^{n}} \bar{\omega}^{p} d \sigma=\int_{S_{+}^{n}} \Theta^{n+1}\left((n+3)\langle S(\tilde{\Theta}), \tilde{\Theta}\rangle-\left\langle S\left(E_{i}\right), E_{i}\right\rangle\right) d \sigma .
$$

\section{Fixed point argument.}

Lemma B.2. for every $p \in \partial \Omega$ and $r$ small, there exit a unique $\hat{\omega}^{p, r}$ and a vector field $\Xi_{p, r}$ on $T_{p} \partial \Omega$ such that

$$
\begin{cases}r H\left(p, r, r \bar{\omega}^{p}+\hat{\omega}\right)=n & \text { in } S_{n}^{+}, \\ \left\langle N_{\partial \Sigma}^{\Sigma}, N_{\partial \Sigma}^{\partial \Omega}\right\rangle=\left\langle\Xi_{p, r}, \tilde{\Theta}\right\rangle & \text { on } S^{n-1} .\end{cases}
$$

Proof. We recall that $\Pi_{1}$ is the $L^{2}$ projection on $\Lambda_{1}$, the space spanned by $\Theta^{i}$, $i=1, \ldots, n$. For any $v \in L^{2}\left(S_{+}^{n}\right)$, we decompose it as

$$
v=\hat{\omega}+\langle\Xi, \tilde{\Theta}\rangle=\omega_{1}+\omega_{0}+\langle\Xi, \tilde{\Theta}\rangle,
$$

where $\Pi_{0} \omega=\omega_{0}=\int_{S_{+}^{n}} \omega d \sigma$ and $\hat{\omega}=\Pi_{1}{ }^{\perp} v$. Recalling the definition of $T$ in (11), we define

$$
\mathscr{L}_{p, r}: \mathscr{C}^{2, \alpha}\left(\overline{S_{+}^{n}}\right) \rightarrow \mathscr{C}^{0, \alpha}\left(\overline{S_{+}^{n}}\right)
$$

by setting

$$
\left\langle\mathscr{L}_{p, r} v, v^{\prime}\right\rangle:=\left\langle\frac{\partial T\left(r, p, r \bar{\omega}^{p}+\omega\right)}{\partial \omega}(p, r, 0)[v], v^{\prime}\right\rangle-\oint_{S^{n-1}}\langle\Xi, \tilde{\Theta}\rangle\left\langle\Xi^{\prime}, \tilde{\Theta}\right\rangle d s,
$$

for all $v^{\prime} \in L^{2}$. By Proposition 3.2,

$$
\begin{aligned}
\left(\mathscr{L}_{p, r} v, v^{\prime}\right)=\int_{S_{+}^{n}}\left(\nabla_{S^{n}} \hat{\omega} \nabla_{S^{n}} \hat{\omega}^{\prime}-n \hat{\omega} \hat{\omega}^{\prime}\right) d \sigma & -\oint_{S^{n-1}}\langle\Xi, \tilde{\Theta}\rangle\left\langle\Xi^{\prime}, \tilde{\Theta}\right\rangle d s \\
& +r \int_{S_{+}^{n}} v^{\prime} L(\hat{\omega}) d \sigma+r \oint_{S^{n-1}} v^{\prime} \bar{L}(\hat{\omega}) d s .
\end{aligned}
$$

Since $\int_{S_{+}^{n}}\left|\nabla \omega_{1}\right|^{2} d \sigma \geq 2(n+1) \int_{S_{+}^{n}}\left|\omega_{1}\right|^{2} d \sigma$, it is easy to see that

$\Pi \circ \mathscr{L}_{p, r} \geq \frac{1}{2}+o_{r}(1), \quad \Pi_{0} \circ \mathscr{L}_{p, r} \leq-n+o_{r}(1), \quad \Pi_{1} \circ \mathscr{L}_{p, r} \leq-\frac{\left|S_{+}^{n}\right|}{n+1}+o_{r}(1)$, 
where $o_{r}(1)$ is a function in $r$ (maybe depending on $p$ ) which tends to zero (uniformly in $p$ ) as $r \rightarrow 0$. From this, we deduce that $\mathscr{L}_{p, r}$ is uniformly invertible and there exists a constant independent of $p$ and $r$ such that

$$
\left\|\mathscr{L}_{p, r}^{-1}\right\|_{L^{2}} \leq C \quad \text { for any } p \in \partial \Omega, r \ll 1 .
$$

Now the system (20) is equivalent to the fixed point equation

$$
v=\left(\mathscr{L}_{p, r}\right)^{-1}\left\{\mathcal{O}_{p}\left(r^{2}\right)+Q_{p}(\hat{\omega})\right\},
$$

where $Q^{2}(\hat{\omega})$ is the quadratic part of the mapping $T$ defined in (11). By elliptic regularity theory, in a small ball of radius $c r^{2}$ in $\Pi_{1} \perp \varphi^{2, \alpha}\left(\overline{S_{+}^{n}}\right) \times T_{p} \partial \Omega$, the above equation has a unique solution $\left(\hat{\omega}^{p, r}, \Xi_{p, r}\right)$ such that (20) is satisfied.

Since the implicit function theorem also applies, one has the smoothness of $p \mapsto \omega^{p, r}$ and $p \mapsto \Xi_{p, r} \in T_{p} \partial \Omega$. Moreover differentiating the mean curvature equation in $p$, using standard elliptic regularity theory, we can deduce that

$$
\left\|\omega^{(\cdot), r}\right\|_{C^{2, \alpha} \times \mathscr{C}^{1}(\partial \Omega)}+\left\|\Xi_{(\cdot), r}\right\|_{\mathscr{C}^{1}(\partial \Omega)} \leq c r^{2}
$$

for some constant $c>0$ independent of $r$.

Variational argument. By Lemma B.2, fixing $r>0$ small, for any $p \in \partial \Omega$, we have a unique hypersurface $\Sigma_{p, r}:=\Sigma_{p, r, \omega^{p, r}}$ which is embedded because the $\mathscr{C}^{1, \alpha}$ bound (up to the boundary) of $\omega^{p, r}:=r \bar{\omega}^{p}+\hat{\omega}^{p, r}$ tends to zero as $r \rightarrow 0$. This now yields for fixed $r>0$ a manifold $\tilde{Z}_{r}$ of sets $E^{p, r} \subset \Omega, p \in \partial \Omega$, bounded by $\Sigma_{p, r}$ and $\partial \Omega$ which is homeomorphic to $\partial \Omega$. We have to show that $\tilde{Z}_{r}$ is a natural constraint for $\mathscr{E}$. For that we define the reduced functional $\varphi_{r}: \partial \Omega \rightarrow \mathbb{R}$ by

$$
\varphi_{r}(p)=\mathscr{E}\left(E^{p, r}\right)=\mathscr{P}_{g}\left(E^{p, r}, \Omega\right)-\frac{n}{r}\left|E^{p, r}\right|_{g},
$$

for any $E^{p, r} \in \tilde{Z}_{r}$.

Lemma B.3. Let $\varphi_{r}$ be given by (21). There exists $r_{0}(\Omega)>0$ such that for any $r \in\left(0, r_{0}\right)$, if $p$ is a critical point of $\varphi_{r}$ then $\Xi_{p, r}=0$.

Proof. Let $p$ be a critical point of $\varphi_{r}$. Then for any vector field $\Xi$ on $T_{p} \partial \Omega$,

$$
d \varphi_{r}(p)[\Xi]=0 .
$$

If $q:=\exp _{p}^{\partial \Omega}(t \Xi)$, then for $t$ sufficiently small the surface $\Sigma_{q, r}$ is a graph over $\Sigma_{p, r}$ for some smooth function $w_{p, r, \Xi, t}$ with variation vector field $\zeta_{p, r, \Xi}$ in $T_{p} \mu$ satisfying

$$
\zeta_{p, r, \Xi}:=\left.\frac{\partial}{\partial t} w_{p, r, \Xi, t}\right|_{t=0} N_{\partial \Sigma}^{\partial \Omega} \quad \text { on } \partial \Sigma_{p, r} \subset \partial \Omega,
$$

where $N_{\partial \Sigma}^{\partial \Omega}$ is the normal of $\partial \Sigma_{p, r}$ in $\partial \Omega$. 
It is easy to see that for any parallel transport, in $\partial \Omega, Z$ along geodesics issued from $p$ of $\Xi$ we have the estimate

$$
\left\|\zeta_{p, r, \Xi}-Z\right\| \leq c r\|\Xi\| \quad \text { on } \partial \Sigma_{p, r} .
$$

Now the first variation of area and volume yield

$$
\begin{aligned}
& 0=d \varphi_{r}(p)[\Xi], \\
& 0=\int_{\Sigma_{p, r}}\left(H_{\Sigma_{p, r}}-\frac{n}{r}\right)\left\langle\zeta_{p, r, \Xi}, N_{\partial \Sigma}^{\Sigma}\right\rangle d \sigma+\oint_{\partial \Sigma_{p, r}}\left\langle\zeta_{p, r, \Xi}, N_{\partial \Sigma}^{\Sigma}\right\rangle d s .
\end{aligned}
$$

By construction,

$$
H_{\Sigma_{p, r}}=\frac{n}{r} \quad \text { in } \Sigma_{p, r} \quad \text { and } \quad\left\langle N_{\partial \Sigma}^{\Sigma}, N_{\partial \Sigma}^{\partial \Omega}\right\rangle=\left\langle\Xi_{p, r}, \widetilde{\Theta}\right\rangle \quad \text { on } \partial \Sigma_{p, r}
$$

thus

We have

$$
\oint_{\partial \Sigma_{p, r}}\left\langle\zeta_{p, r, \Xi}, N_{\partial \Sigma}^{\partial \Omega}\right\rangle\left\langle\Xi_{p, r}, \widetilde{\Theta}\right\rangle d s=0
$$

$$
\left\langle\zeta_{p, r, \Xi}, N_{\partial \Sigma}^{\partial \Omega}\right\rangle=-\langle Z, \tilde{\Upsilon}\rangle+\left\langle\zeta_{p, r, \Xi}-Z, N_{\partial \Sigma}^{\partial \Omega}\right\rangle+\left\langle Z, N_{\partial \Sigma}^{\partial \Omega}+\tilde{\Upsilon}\right\rangle \quad \text { on } \partial \Sigma_{p, r} .
$$

The expansions of the metric together with the normal $N_{\partial \Sigma}^{\partial \Omega}$ (see (8) and (9)) show that

$$
N_{\partial \Sigma}^{\partial \Omega}+\tilde{\Upsilon}=O(r) \quad \text { while } \quad \tilde{\Upsilon}=\widetilde{\Theta}(1+O(r)) .
$$

Therefore we have the estimates

$$
\left|\left\langle\zeta_{p, r, \Xi}, N_{\partial \Sigma}^{\partial \Omega}\right\rangle+\langle\Xi, \widetilde{\Theta}\rangle\right| \leq c r\|\Xi\| .
$$

This implies, also by Hölder inequality, that

$$
\begin{aligned}
\oint_{\partial \Sigma_{p, r}}\left\langle\Xi_{p, r}, \widetilde{\Theta}\right\rangle\langle\Xi, \widetilde{\Theta}\rangle d s & \leq c r\|\Xi\| \oint_{\partial \Sigma_{p, r}}\left\langle\Xi_{p, r}, \widetilde{\Theta}\right\rangle d s \\
& \leq c r\|\Xi\|\left(\oint_{\partial \Sigma_{p, r}} d s\right)^{1 / 2}\left(\oint_{\partial \Sigma_{p, r}}\left|\left\langle\Xi_{p, r}, \widetilde{\Theta}\right\rangle\right|^{2} d s\right)^{1 / 2} .
\end{aligned}
$$

Using the expansion of the metric of small perturbed geodesic sphere (see [Pacard and $\mathrm{Xu} 2009$, Lemma 2.1]) we find that

$$
\oint_{\partial \Sigma_{p, r}}\left\langle\Xi_{p, r}, \widetilde{\Theta}\right\rangle\langle\Xi, \widetilde{\Theta}\rangle d s \leq c r\|\Xi\| r^{(n-1) / 2}\left(\oint_{\partial \Sigma_{p, r}}\left|\left\langle\Xi_{p, r}, \widetilde{\Theta}\right\rangle\right|^{2} d s\right)^{1 / 2},
$$

while

$$
\frac{1}{2} \operatorname{Area}\left(S^{n-1}\right) r^{n-1}\|\Xi\|^{2} \leq n \oint_{\partial \Sigma_{p, r}}|\langle\Xi, \widetilde{\Theta}\rangle|^{2} d s .
$$


Hence we have

$$
\oint_{\partial \Sigma_{p, r}}\left\langle\Xi_{p, r}, \widetilde{\Theta}\right\rangle\langle\Xi, \widetilde{\Theta}\rangle d s \leq c r\left(\oint_{\partial \Sigma_{p, r}}|\langle\Xi, \widetilde{\Theta}\rangle|^{2} d s\right)^{1 / 2}\left(\oint_{\partial \Sigma_{p, r}}\left|\left\langle\Xi_{p, r}, \widetilde{\Theta}\right\rangle\right|^{2} d s\right)^{1 / 2} .
$$

And, finally setting $\Xi=\Xi_{p, r}$, we obtain

$$
\oint_{\partial \Sigma_{p, r}}\left|\left\langle\Xi_{p, r}, \widetilde{\Theta}\right\rangle\right|^{2} d s \leq c r \oint_{\partial \Sigma_{p, r}}\left|\left\langle\Xi_{p, r}, \widetilde{\Theta}\right\rangle\right|^{2} d s .
$$

Consequently $\Xi_{p, r}=0$ for $r$ small.

Using Lemmas A.3 and A.4 in Appendix A, we get

$$
\begin{aligned}
& r^{-n} \mathscr{P}_{g}\left(E_{p, r}, \Omega\right)=\mathscr{P}\left(B^{n+1}, \mathbb{R}_{+}^{n+1}\right)+r \int_{S_{+}^{n}}\left(\left\langle S\left(E_{i}\right), E_{i}\right\rangle\right.-\langle S(\widetilde{\Theta}), \widetilde{\Theta}\rangle) \Theta^{n+1} d \sigma \\
&+n \int_{S_{+}^{n}} \omega d \sigma+O_{p}\left(r^{2}\right), \\
& r^{-1-n}\left|E_{p, r}\right|_{g}=\frac{1}{n+1} \mathscr{P}\left(B^{n+1}, \mathbb{R}_{+}^{n+1}\right)+\frac{r}{n+2}\left\langle S\left(E_{i}\right), E_{i}\right\rangle \int_{S_{+}^{n}} \Theta^{n+1} d \sigma \\
&+\int_{S_{+}^{n}} \omega d \sigma+O_{p}\left(r^{2}\right) .
\end{aligned}
$$

This now gives (recalling (14))

$$
\begin{aligned}
r^{-n} \varphi_{r}(p)= & r^{-n} \mathscr{E}_{r}\left(E^{p, r}\right) \\
= & \frac{1}{n+1} \mathscr{P}\left(B^{n+1}, \mathbb{R}_{+}^{n+1}\right) \\
\quad & \quad+r \int_{S_{+}^{n}}\left(\frac{2}{n+2}\left\langle S\left(E_{i}\right), E_{i}\right\rangle-\langle S(\widetilde{\Theta}), \widetilde{\Theta}\rangle\right) \Theta^{n+1} d \sigma+O_{p}\left(r^{2}\right) \\
= & \frac{1}{n+1} \mathscr{P}\left(B^{n+1}, \mathbb{R}_{+}^{n+1}\right)-\frac{n\left|B^{n}\right|}{(n+2)} r H_{\partial \Omega}(p)+O_{p}\left(r^{2}\right) .
\end{aligned}
$$

We end the proof of Proposition B.1 by setting

$$
f(r, p):=\frac{-(n+2)}{r n\left|B^{n}\right|}\left(r^{-n} \varphi(p)-\frac{1}{n+1} \mathscr{P}\left(B^{n+1}, \mathbb{R}_{+}^{n+1}\right)\right)=H_{\partial \Omega}(p)+O_{p}(r) .
$$

Using (19), we also get precise expansions of the area of the of constant mean curvature hypersurfaces we have constructed as well as the volume of the domain they enclose.

Corollary B.4. For any $E^{p, r} \in \tilde{Z}_{r}$,

$$
\begin{gathered}
r^{-n} \mathscr{P}_{g}\left(E^{p, r}, \Omega\right)=\mathscr{P}\left(B^{n+1}, \mathbb{R}_{+}^{n+1}\right)-n\left|B^{n}\right| r H_{\partial \Omega}(p)+O_{p}\left(r^{2}\right), \\
r^{-n-1}\left|E^{p, r}\right|_{g}=\left|B_{+}^{n+1}\right|-\frac{n+1}{n+2}\left|B^{n}\right| r H_{\partial \Omega}(p)+O_{p}\left(r^{2}\right) .
\end{gathered}
$$




\section{Acknowledgments}

The author wishes to thank Professor Andrea Malchiodi for useful discussions.

\section{References}

[Ambrosetti and Badiale 1998] A. Ambrosetti and M. Badiale, "Variational perturbative methods and bifurcation of bound states from the essential spectrum", Proc. Roy. Soc. Edinburgh Sect. A 128:6 (1998), 1131-1161. MR 2000a:34035 Zbl 0928.34029

[Ambrosetti and Malchiodi 2006] A. Ambrosetti and A. Malchiodi, Perturbation methods and semilinear elliptic problems on $\mathbf{R}^{n}$, Progress in Math. 240, Birkhäuser, Basel, 2006. MR 2007k:35005 Zbl 1115.35004

[Bayle and Rosales 2005] V. Bayle and C. Rosales, "Some isoperimetric comparison theorems for convex bodies in Riemannian manifolds", Indiana Univ. Math. J. 54:5 (2005), 1371-1394. MR 2006f:53040 Zbl 1085.53025

[Bérard and Meyer 1982] P. Bérard and D. Meyer, "Inégalités isopérimétriques et applications", Ann. Sci. École Norm. Sup. (4) 15:3 (1982), 513-541. MR 84h:58147 Zbl 0527.35020

[Bürger and Kuwert 2008] W. Bürger and E. Kuwert, "Area-minimizing disks with free boundary and prescribed enclosed volume", J. Reine Angew. Math. 621 (2008), 1-27. MR 2431248 Zbl 05344055

[Chavel 1984] I. Chavel, Eigenvalues in Riemannian geometry, Pure and Applied Math. 115, Academic Press, Orlando, FL, 1984. MR 86g:58140 Zbl 0551.53001

[Chavel 2001] I. Chavel, Isoperimetric inequalities. Differential geometric and analytic perspectives, Cambridge Tracts in Math. 145, Cambridge Univ. Press, 2001. MR 2002h:58040 Zbl 0988. 51019

[Choe et al. 2007] J. Choe, M. Ghomi, and M. Ritoré, "The relative isoperimetric inequality outside convex domains in $\mathbf{R}^{n}$ ", Calc. Var. Partial Differential Equations 29:4 (2007), 421-429. MR 2008k: 58042 Zbl 1116.58016

[Druet 2002] O. Druet, "Sharp local isoperimetric inequalities involving the scalar curvature", Proc. Amer. Math. Soc. 130:8 (2002), 2351-2361. MR 2003b:53036 Zbl 1067.53026

[Fall 2007] M. M. Fall, "Embedded disc-type surfaces with large constant mean curvature and free boundaries", SISSA Preprint, 2007, Available at https://digitallibrary.sissa.it/retrieve/4435/ CMC-surfaces-3.pdf. To appear in Commun. Contemp. Math.

[Fall and Mahmoudi 2008] M. M. Fall and F. Mahmoudi, "Hypersurfaces with free boundary and large constant mean curvature: concentration along submanifolds", Ann. Sc. Norm. Super. Pisa Cl. Sci. (5) 7:3 (2008), 407-446. MR MR2466435

[Grüter 1987] M. Grüter, "Regularity results for minimizing currents with a free boundary", J. Reine Angew. Math. 375/376 (1987), 307-325. MR 88d:49040 Zbl 0618.35041

[Grüter and Jost 1986] M. Grüter and J. Jost, "On embedded minimal disks in convex bodies", Ann. Inst. H. Poincaré Anal. Non Linéaire 3:5 (1986), 345-390. MR 88f:49029 Zbl 0617.49017

[Jost 1986] J. Jost, "Existence results for embedded minimal surfaces of controlled topological type. I”, Ann. Scuola Norm. Sup. Pisa Cl. Sci. (4) 13:1 (1986), 15-50. MR 89m:58040 Zbl 0619.49019

[Kapouleas 1991] N. Kapouleas, "Compact constant mean curvature surfaces in Euclidean threespace”, J. Differential Geom. 33:3 (1991), 683-715. MR 93a:53007b Zbl 0727.53063

[Morgan 1988] F. Morgan, Geometric measure theory. A beginner's guide, Academic Press, Boston, 1988. MR 89f:49036 Zbl 0671.49043 
[Morgan and Johnson 2000] F. Morgan and D. L. Johnson, "Some sharp isoperimetric theorems for Riemannian manifolds", Indiana Univ. Math. J. 49:3 (2000), 1017-1041. MR 2002e:53043 Zbl 1021.53020

[Nardulli 2006] S. Nardulli, "Régularité des solutions du problème isopérimétrique proches de variétés lisses”, Preprint, Université de Paris Sud, 2006.

[Nardulli 2007] S. Nardulli, "The isoperimetric profile of a compact Riemannian manifold for small volumes", Preprint, University of Paris Sud (Orsay), 2007. arXiv 0710.1396v1

[Pacard and Xu 2009] F. Pacard and X. Xu, "Constant mean curvature spheres in Riemannian manifolds”, Manuscripta Math. 128:3 (2009), 275-295. MR MR2481045

[Ritoré and Rosales 2004] M. Ritoré and C. Rosales, "Existence and characterization of regions minimizing perimeter under a volume constraint inside Euclidean cones", Trans. Amer. Math. Soc. 356:11 (2004), 4601-4622. MR 2005g:49076 Zbl 1057.53023

[Ros 2005] A. Ros, "The isoperimetric problem", pp. 175-209 in Global theory of minimal surfaces, edited by D. Hoffman, Clay Math. Proc. 2, Amer. Math. Soc., Providence, RI, 2005. MR2006e: 53023 Zbl 1125.49034

[Ros and Vergasta 1995] A. Ros and E. Vergasta, "Stability for hypersurfaces of constant mean curvature with free boundary”, Geom. Dedicata 56:1 (1995), 19-33. MR 96h:53013 Zbl 0912.53009

[Sternberg and Zumbrun 1998] P. Sternberg and K. Zumbrun, "Connectivity of phase boundaries in strictly convex domains”, Arch. Rational Mech. Anal. 141:4 (1998), 375-400. MR 99c:49045 Zbl 0911.49025

[Struwe 1984] M. Struwe, “On a free boundary problem for minimal surfaces”, Invent. Math. 75:3 (1984), 547-560. MR 85a:58019 Zbl 0537.35037

[Struwe 1988] M. Struwe, "The existence of surfaces of constant mean curvature with free boundaries”, Acta Math. 160:1-2 (1988), 19-64. MR 89a:53012 Zbl 0646.53005

Received October 1, 2008.

\section{Mouhamed Moustapha Fall}

SISSA

VIA BEIRUT 2-4

34151 TRIESTE

ITALY

fall@sissa.it 